\title{
Einleitung
}

\author{
Science and Technology Studies - \\ Wissenschafts- und Technikforschung aus sozial- und \\ kulturanthropologischer Perspektive
}

Jörg NiewÖHner, Estrid Sørensen, Stefan BeCK

Wissenschaft, wissenschaftliches Wissen und Technologien sind allgegenwärtig in den Alltagen westlich-moderner Gesellschaften. Sie leisten entscheidende Beiträge zu gesellschaftlichen Ordnungsprozessen und formen damit unsere Selbstverständnisse und unser Zusammenleben. Gleichzeitig verläuft diese Wirkkette aber auch andersherum. Gesellschaft formt Wissensproduktion und Technologieentwicklung. Wissenschaft vollzieht sich in Alltagen und diese Alltage in Laboren, in Designstudios, in Krankenhäusern oder in Unternehmen sind immer auch vielfältig geprägt von den sozialen, kulturellen, politischen und ökonomischen Kontexten, in denen sie sich entfalten. Aus einer sozial- und kulturwissenschaftlichen Perspektive auf Alltagsleben sind Wissenschaft, Technologie und Gesellschaft also untrennbar miteinander verknüpft.

Es ist eine zentrale Aufgabe des Forschungsfelds der Science and Technology Studies, diese Verschränkung von Wissenschaft, Technologie und Gesellschaft im Alltag zu untersuchen und damit unter anderem auch die Rolle von Wissen und Technologie in gesellschaftlichen Ordnungsprozessen näher zu bestimmen. Konkret bedeutet dies, dass in empirischen Studien vornehmlich solche Felder untersucht werden, in denen verschiedene Wissensformen und Technologien um Deutungshoheit und Wirkmacht konkurrieren; in denen sie stabilisierend oder irritierend wirken; in denen Wis- 
sen und Technologien weiterentwickelt werden oder in Vergessenheit geraten; in denen Wissen und technische Artefakte im Gebrauch beobachtet werden können; und in denen entscheidende Veränderungen der gegenwärtigen Ordnungsprozesse entweder Wissenschaft und Technologieentwicklung entscheidend beeinflussen oder durch solche angetrieben werden.

Dieser Band konzentriert sich auf ein Teilgebiet der Science and Technology Studies (STS), namentlich STS in seiner sozialanthropologischen Ausrichtung. Dies bedeutet, dass zum einen vor allem solche Forschungsansätze vorgestellt werden, die sich auf das Theorie- und Methodenrepertoire der internationalen Sozial- und Kulturanthropologie beziehen bzw. von diesem inspiriert sind. Zum anderen heißt es, dass disziplinübergreifende Forschungsansätze, wie z. B. die Akteur-Netzwerk Theorie, immer aus sozial- und kulturanthropologischer Perspektive diskutiert werden. Aus dieser Auswahl ergibt sich ein besonderes Interesse der folgenden Kapitel an:

- praxistheoretischen Forschungsansätzen, die Alltag in den Vordergrund stellen und ethnographisch vorgehen;

- Fragestellungen, die einen Bezug zu wichtigen, Theorie-prägenden Figuren aus der sozial- und kulturanthropologischen Fachgeschichte und -diskussion herstellen, vor allem zu Fragen nach Rationalität, Materialität, Wissen und Praxis;

- Veränderungen im Verhältnis von Natur und Kultur und damit auch Veränderungen in den Konstitutionsprozessen von Individualität und Sozialität, sowie schließlich

- Forschungsfeldern, deren Untersuchung das Potential birgt, wichtige Impulse in die Sozial- und Kulturanthropologie zurückzugeben, wie es vielleicht in den letzten Jahren am deutlichsten im Feld der Reproduktionsmedizin und der neuen Verwandtschaftsethnologie geworden ist. (z. B. Beck et al. 2007)

Mit dieser Setzung ist selbstverständlich keine Wertung intendiert. Sie reflektiert die Forschungsinteressen und Prioritätensetzungen der Herausgeberinnen und Herausgeber wie auch der Autorinnen und Autoren, die zum Zeitpunkt der Erstellung dieses Bandes von 2009 bis 2011 allesamt am Institut für Europäische Ethnologie der Humboldt-Universität zu Berlin gearbeitet haben. 
Die folgenden einleitenden Abschnitte gliedern sich in drei Teile. Zunächst wird das Forschungsfeld der Science and Technology Studies in einem kurzen, chronologisch aufgebauten, thematischen Abriss skizziert, um den Leserinnen und Lesern, die mit dem Forschungsfeld bisher noch gar nicht in Berührung gekommen sind, einen ersten Einstieg zu ermöglichen. Ein solcher Versuch, das Forschungsfeld quasi neutral mit einem »view from nowhere« (Nagel 1986) zu beschreiben, widerspricht teilweise den zentralen Anliegen der Wissenschafts- und Technikforschung, die sich immer um die Analyse von Forschung und Wissen als historisch wie sozial situiert bemühen. Ein solches Vorgehen erschien uns in diesem Kontext aber aus didaktischen Gründen unvermeidlich. Dieser allgemeinen Einführung folgt ein zweiter Teil, der in einiger Ausführlichkeit die sozialanthropologische Perspektivierung dieses Bandes näher erläutert. Leserinnen und Leser, die mit den Grundzügen des Forschungsfelds vertraut sind, finden hier einen Einstieg in die Spezifik dieses Bandes. Abschließend werden in einem kurzen dritten Teil die Struktur dieses Bandes vorgestellt und einige didaktische Hilfestellungen zu den Kapiteln näher erläutert.

\section{VIEW FROM NOWHERE}

\section{Was sind die Fragestellungen der Science and Technology Studies?}

Science and Technology Studies bezeichnet ein hochgradig transdisziplinäres Forschungsfeld. Sein vorrangiges Ziel ist die empirische Untersuchung der vielfältigen Rollen von Wissen und Technologie in modernen Gesellschaften unter Verwendung von Methoden der Sozial-, Kultur- und Geschichtswissenschaften.

Das Forschungsfeld der STS ist in den späten 1970er Jahren vor allem am Schnittfeld von Wissenschaftsphilosophie, -geschichte und -soziologie auf der einen und Technikphilosophie, -geschichte und -soziologie auf der anderen Seite entstanden. Zentrales Anliegen war es, wissenschaftliches Wissen und Technik als soziale und kulturelle Phänomene zu verstehen und einer kritischen Analyse zugänglich zu machen. Ausgangspunkt war dabei die Beobachtung zahlreicher Forscherinnen und Forscher, dass erstens Wissenschaft und Technologie moderne Lebensweisen zwar zutiefst prägen, diese Prägungen von Handeln und Denken, von Politik und Ökonomie, von 
menschlichen Selbstverständnissen und moralischen Ordnungen jedoch nur unzureichend analysiert wurden. Zweitens wurde Wissenschaft zunehmend als eine Institution und Praxis verstanden, die ihrerseits in relevanter Weise durch Gesellschaft, d. h. durch lokale soziale und historische Konfigurationen geprägt ist. Und drittens wuchs die Unzufriedenheit mit hierarchischen Verständnissen verschiedener Wissensformen, die wissenschaftliches Wissen über oder zumindest abseits von anderen »alltäglichen« Wissensformen positionierten.

Die kritische Reflexion von Wissenschaft war bis dahin vorwiegend als Domäne der Philosophie angesehen worden, und den Geschichtswissenschaften blieb es vorbehalten, die Entwicklung technischer Innovationen nachzuvollziehen. Vor allem im Feld der Wissenschaftsforschung wurden Fragen nach Ontologie und Epistemologie, d. h. danach, was und wie Phänomene sind und wie wir dies wissen können, lange Zeit nicht empirisch mit Blick auf wissenschaftliche Praxis untersucht. Der Alltag von Wissenschaft spielte keine Rolle. Stattdessen wurde entweder theoretisch, innerhalb etablierter logischer oder rationaler Denkmodelle vorgegangen oder anhand von historischen Fallbeispielen, die anekdotisch oder mittels Quellenanalyse erschlossen wurden. (WissenschaftstheORIE) ${ }^{1}$ Auch die Wissenschaftssoziologie konzentrierte sich Mitte der 1950er und 1960er Jahre nicht auf Fragen nach alltäglichem Wissenschaftshandeln und wissenschaftlichem Wissen, sondern rückte Wissenschaft als Institution in den Vordergrund. (WISSENSCHAFTSSOZIOLOGIE)

Das Forschungsfeld der STS hat daher die alte Aufgabenteilung zwischen den Disziplinen grundlegend verändert und begonnen, sich Fragen von Wissensproduktion und seinen epistemologischen Voraussetzungen wie Konsequenzen empirisch zu widmen. Die Kombination der Perspektiven auf Wissenschaft und Technologie ermöglichte neue Einsichten - etwa dadurch, dass der Beitrag von Apparaten, Aufzeichnungsinstrumenten und Maschinen für die Produktion wissenschaftlichen Wissens genauer untersucht wurde. Die Frage, wie wir etwas wissen und wie Erkenntnis entsteht, war damit Ende der 1970er Jahre nicht länger lediglich eine abstrakt zu erörternde Frage nach geistigen Prozessen, sondern auch eine empirisch zu beantwortende Frage nach konkreter Forschungspraxis. Wissenschaft er-

1 Begriffe oder Phrasen in Kapitälchen verweisen immer auf das entsprechende Kapitel in diesem Band. 
schien in diesen Studien nicht mehr nur als Ergebnis rein intellektueller kontemplativer Tätigkeit, sondern als Ergebnis praktischen Tuns und sozialen Handelns. Dieser Wandel brachte mit der Soziologie wissenschaftlichen Wissens zunächst vor allem die verschiedenen Interessen der Forschenden ins Zentrum der Untersuchungen, die bis dahin bestenfalls als Störfaktor oder Barriere für wirkliche Erkenntnis thematisiert und damit de facto ausgeblendet worden waren. (Sociology OF ScIENTIFIC KNOwLEDGE) Rasch kamen mit den so genannten Laborstudien ethnographische Untersuchungen verschiedener Forschungsalltage in Laboren hinzu. (LABORSTUDIEN) Auch die Untersuchungen von Technologie und Technologienutzung und -entwicklung reichten bald über die philosophischen und sozial- und kulturtheoretischen Analysen von Technik, als determinierendem und antihumanistischem Phänomen, hinaus. Stattdessen setzte sich ein empirischer Zugriff durch, der Technologie stets im Kontext seiner Produktions- und Nutzungspraxen begreift - ob in Wissenschaft oder Alltag. (SOZIALE KONSTRUKTION VON TECHNOLOGIE)

In der frühen STS Forschung, in den 1980er Jahren, standen vor allem Formen und Orte der Wissensproduktion und der Technologieentwicklung im Zentrum der Aufmerksamkeit. Durch Analysen dieser Zentren von wissenschaftlicher Wissensproduktion, gewissermaßen dem säkularen Hort von Fortschritt und Modernisierung der westlichen Welt, gelang es STS, wissenschaftliches Wissen und Technologie sozialwissenschaftlichen Analysen zugänglich zu machen. Im Rückblick erscheint dies für die heutigen Sozial- und Kulturwissenschaften mehr als selbstverständlich; Anfang der 1980er Jahre hatte dies jedoch durchaus ein provokatives Potential. Denn die sozialkonstruktivistische Wende entzauberte wissenschaftliches Wissen und ließ seinen epistemologischen Sonderstatus verblassen. ${ }^{2}$ Wissen jeder Art gilt heute Vielen in den Sozial- und Kulturwissenschaften lediglich als das profane Ergebnis eines hochgradig kontingenten sozialen Prozesses; wissenschaftliches Wissen wird damit prinzipiell genauso behandelt, wie andere Wissenstypen auch, z. B. Alltagswissen. Seine Besonderheit liegt nun in seinen spezifischen Produktionsbedingungen,

2 Dabei sei angemerkt, dass es sich hier um eine Entzauberung von Wissenschaft selbst handelt und nicht, wie noch bei Max Weber, um eine Entzauberung durch Wissenschaft. (Weber 1922) 
seinem - kulturell zertifizierten - Sonderstatus und seiner herausgehobenen Funktion als Mittel der »gesellschaftlichen Selbsteinwirkung«, etwa bei der Entwicklung technischer Innovationen oder als Reflexionsinstrument. Die Science and Technology Studies haben entscheidend zu diesem veränderten Blick auf Wissen beigetragen.

Jedoch wurde schon früh deutlich, spätestens in den späten 1980er Jahren, dass die sozialkonstruktivistische Wende zwar wissenschaftliches Wissen entzaubert hatte, dass damit allerdings keineswegs drängende Fragen nach den vielfältigen Rollen wissenschaftlichen Wissens in den Ordnungsprozessen moderner Gesellschaften beantwortet waren. Zwei Entwicklungen, die eng mit den Forschungen der Science and Technology Studies assoziiert sind, haben in dieser Zeit ihren Ursprung. Erstens beginnt die Akteur-Netzwerk Theorie jenseits von sozialkonstruktivistischer Kritik, die für die Moderne kennzeichnende Trennung von Natur und Kultur in Frage zu stellen und sie durch netzwerkorientierte und relationale Analysen wenn nicht aufzuheben, so doch für wichtige Zusammenhänge zu suspendieren. (AKTEUR-NETZWERK THEORIE) Dieser Schritt vollzieht sich in theoretischer Nähe zu einer in den 1980er Jahren an Bedeutung gewinnenden, zweiten Welle feministischer Kritik, die vor allem die US amerikanische Kulturanthropologie signifikant beeinflusst hat. Und Wissenschaftlerinnen aus diesem Feld der Kulturanthropologie waren es auch, die in einer viel beachteten Entwicklung ein anthropologisch orientiertes, theoretisches Programm für das Feld der Science and Technology Studies entworfen haben, das das Feld bis heute herausfordert. Wissenschaft, so ihre Argumentation, solle als integraler Bestandteil von Kultur und gesellschaftlichen Machtverhältnissen verstanden werden und nicht als wie auch immer geartete außerkulturelle oder vorkulturelle Praxisform. Dieser Ansatz formuliert vor allem den Anspruch, Wissen und Technologie nicht nur in seinen (Produktions-)Zentren abseits von gesellschaftlichem Alltag zu untersuchen, sondern als Bestandteil des Alltags. Damit wird wissenschaftliches Wissen in einem Kontinuum von Wissenspraxen verortet und zugleich werden fundamentale Unterscheidungen, etwa zwischen »Expertin« und »Laie«, hinterfragt. Wissen wird im Rahmen dieser Entwicklungen der 1990er Jahre zu einer materiellsemiotischen Praxis, die historisch, sozial, kulturell und epistemisch situiert analysiert werden soll. 
Theoretisch wie empirisch geht das Forschungsfeld der Science and Technology Studies damit in seinen Erklärungs- und Kritikansprüchen wesentlich über die Erforschung von Wissenschaft und Technologieentwicklung hinaus. Stattdessen etabliert es sich als ein Ansatz, der die vielfältigen Rollen von Wissen und Technologie - immer verstanden und analysiert als eingebettet in Praxis - in gesellschaftlichen Ordnungsprozessen empirisch untersucht, historisch situiert und auf ihre epistemologischen und ontologischen Auswirkungen hin problematisiert und theoretisiert. In dieser Ausrichtung stellt das Forschungsfeld der Science and Technology Studies eine wichtige Forschungsrichtung in der internationalen Sozial- und Kulturanthropologie und damit auch der Europäischen Ethnologie dar.

Aktuelle Forschungsfelder der STS schließen dabei an Themen an, die in der internationalen social-cultural anthropology und der Europäischen Ethnologie teilweise eine lange Tradition haben. Die Analyse von aktuellen Entwicklungen in den Lebenswissenschaften und der Biomedizin etwa stellt fruchtbare Bezüge zu den Arbeiten der Medizinanthropologie her, Forschungen zur Nutzung von neuen Medien und ihren Konsequenzen können Untersuchungen des Faches zur Massenkultur und der neueren media anthropology aufgreifen. Studien zur ökologischen Anthropologie aus den 1950er und 60er Jahren können theoretische wie methodologische Hinweise für aktuelle Untersuchungen des Klimawandels mit seinen wichtigen Implikationen für Lebensstile, Landnutzung und Siedlungs- und Migrationsbewegungen sowie den damit befassten wissenschaftlichen Kontroversen bieten. Eine solche, durch historische und vergleichende Analysen angereicherte Perspektive kann produktiv gemacht werden für die Untersuchung der Steuerbarkeit von Innovation und ihrer Bedeutung für wirtschaftliche, politische und soziale Entwicklungen. ${ }^{3}$ Allen diesen Feldern ist gemein, dass in ihnen Wissensproduktion nie abseits von oder gar als determinierend für gesellschaftliche Kontexte und Entwicklungen untersucht wird, sondern immer als wichtiger Bestandteil von vielfältigen Wechselwirkungen zwischen verschiedenen gesellschaftlichen Feldern.

3 Beispiele für Studien dieser Art aus den Arbeiten des »Labors: Sozialanthropologische Wissenschafts- und Technikforschung« am Institut für Europäische Ethnologie der Humboldt-Universität zu Berlin finden sich u.a. hier: Niewöhner, et al. 2011; Beck, et al. 2007; Mathar 2010; Scheffer and Niewöhner 2010; Kontopodis, et al. 2011; Niewöhner, et al. 2011a; Mathar and Jansen 2010; Niewöhner, et al. 2008. 


\section{Wie wird in den Science and Technology Studies geforscht?}

Das Methodenspektrum in den Science and Technology Studies ist sehr breit und reicht von quantitativen Verfahren beispielsweise zur Analyse von Innovationsprozessen, über historische Quellenanalysen und verschiedene Interviewtypen bis zu teilnehmender Beobachtung in einzelnen Feldern über lange Zeiträume hinweg. Dabei orientiert sich die Methodenauswahl, wie in Wissenschaft üblich, primär an den zentralen Forschungsfragen und Forschungsfeldern der je konkreten Projekte. Da es sich bei den Science and Technology Studies um ein transdisziplinäres Forschungsfeld handelt, kommt allerdings hinzu, dass die Forschungsfragen und die Perspektiven auf Forschungsfelder in den meisten Fällen zumindest »Rückstände« von disziplinären Orientierungen - etwa der Soziologie, Anthropologie, Psychologie oder Politikwissenschaften - aufweisen und mit diesen auch immer methodische Präferenzen einhergehen. Ein methodischer Kanon, im Sinne eines verbindlich gemachten Methodenspektrums, lässt sich demnach nicht ausmachen und Forschende, die sich in dieses Forschungsfeld einarbeiten, tun gut daran, sich eine methodische Offenheit zu erhalten.

Es sei hier ebenfalls herausgestellt, dass in den Science and Technology Studies Methoden immer als zentraler Teil von Wissenspraxis verstanden werden. Methoden sind nicht einfach Werkzeug, um eine Wirklichkeit »da draußen« mehr oder weniger genau abzulesen. Vielmehr tragen Methoden zu spezifischen Konfigurierungen von Realität bei. Forschende legen daher zum einen Wert auf das Transparent-Machen des methodischen Zugriffs; zum anderen werden Art und Reichweite von Wissen nie abseits der Methoden diskutiert, mit denen es produziert wurde. Unterschiedliche Methoden produzieren unterschiedliche Formen von Wissen. Methoden haben daher in den Science and Technology Studies argumentativen Charakter und sind nicht nur Mittel zum Zweck.

\section{Interviews und Diskursanalyse}

Trotz dieser Offenheit lassen sich Schwerpunkte und Präferenzen ausmachen, von denen die folgenden Abschnitte drei herausgreifen. Begonnen haben die Science and Technology Studies in ihrer soziologischen Orientierung mit qualitativen Interviews mit Forschenden in der Physik. 
Die im Entstehen begriffene Soziologie wissenschaftlichen Wissens (SoCIOLOGY OF SCIENTIFIC KNOWLEDGE) hatte es sich zum Ziel gesetzt, die Asymmetrie in den damaligen wissenschaftstheoretischen Untersuchungen zu beheben. Die Wissenschaftstheorie, vor allem in ihrer historistischen und retrospektiven Prägung im Stile eines Thomas Kuhn, hatte den Bedingungen von Erkenntnis, wie sie sich für die Wissenschaftler in der Situation der Wissensproduktion stellten, bis dahin nur insofern Beachtung geschenkt, als dass sich mit ihnen Irrtümer und Abweichungen vom geraden Weg des wissenschaftlichen Fortschritts im positivistischen Sinne erklären ließen. Es herrschte vielmehr die Annahme, dass erfolgreiches - und als "wahr« gesehenes - wissenschaftliches Wissen nicht aus seinen sozialen Entstehungskontexten erklärt werden musste, da es sich dem systematischen Einsatz von Methoden und rationalen Theorien verdankte, die keiner besonderen Nachfrage bedurften. (WISSENSCHAFTSTHEORIE)

Hieran wurde aus zwei Richtungen Kritik geübt, die beide »Interesse« als wesentliches Moment wissenschaftlicher Praktiken thematisierten, dabei jedoch sehr unterschiedlich ansetzten: Die Kritische Theorie der Frankfurter Schule versuchte mit dem Begriff des »erkenntnisleitenden Interesses« herauszuarbeiten, dass die in der Wissenschaft geltenden Normen und Praktiken, mit denen eigene Objektivitätsansprüche gegen Subjektivität und partikulare Interessen, gegen religiöse Doktrinen und weltliche Herrschaftsansprüche verteidigt wurden, selbst nicht kritisch reflektiert würden. Erst die systematische, kulturhistorisch informierte Analyse der menschlichen Erkenntnisfähigkeit, die stets an Selbsterhaltungsinteressen der in Gesellschaften lebenden Menschen gebunden sei, erlaube es zu erkennen, dass "wissenschaftliche Objektivität« stets sozial und kulturell imprägniert sei: »erkenntnisleitende Interessen bilden sich im Medium von Arbeit, Sprache und Herrschaft«, womit auch Naturerkenntnis stets relativ zu gesellschaftlichen Formationen sei. (Habermas 1969: 163)

Während die Kritische Theorie ausgehend von einem philosophischanthropologischen Argument die Objektivitätsansprüche der Wissenschaft durch das Konzept des »erkenntnisleitenden Interesses« grundsätzlich zu entzaubern suchte und daraus eine Kritik von Wissenschaft und Technologie als »Ideologie« westlicher Gesellschaften ableitete, ging die Sociology of Scientific Knowledge, wie sie in Großbritannien entwickelt wurde, empirischer vor. Auch sie ging davon aus, dass alles Wissen sozial konstruiert sei, auch jenes, dass als »wahr« angesehen wurde - auch »wahres Wissen« 
könne nicht beanspruchen, unvermittelt Natur zu repräsentieren. Notwendig sei daher eine symmetrische Analyse von "wahrem» wie »falschem» Wissen in Bezug auf seine jeweiligen Konstruktionsbedingungen und -prozesse. (Bloor 1976) So entwickelte diese Forschungsrichtung zunächst einen sozialwissenschaftlichen Zugang, im Besonderen zu den Interessen der führenden Wissenschaftler, z. B. in Teilgebieten der Physik. (Collins 1975) Um diese Interessen zu untersuchen, brachten die Forschenden erstmals in der Geschichte der Untersuchung von Wissenschaft systematisch qualitative Interviews zum Einsatz. Damit war die Analyse der Produktionsbedingungen von wissenschaftlichem Wissen dem Monopol der Philosophie und Wissenschaftstheorie entzogen und der Sozialforschung zugänglich gemacht worden.

Verschiedene Formen des semi-strukturierten Interviews und der qualitativen Analyse dieses Materials haben sich bis heute als die wohl wichtigste Form der Datenerhebung in den Science and Technology Studies gehalten. Dabei geht es heute meist nicht mehr, oder zumindest nicht mehr nur, um die Interessen von einzelnen Forschenden, sondern um ein breites Themenspektrum von Forschungsinfrastrukturen, epistemologischen Konsequenzen von Forschungsansätzen oder Auswirkungen auf benachbarte Felder und Praxiskontexte sowie gesellschaftliche Alltage. Angelehnt an die Arbeiten des französischen Sozialtheoretikers und Philosophen Michel Foucault zur Mikrophysik der Macht und der engen Verschränkung von Macht und Wissen in der Herstellung und Aufrechterhaltung von Wahrheitsdiskursen, werden heute häufig qualitative Interviews mit Schlüsselfiguren in den jeweiligen Forschungsfeldern mit Analysen der wissenschaftlichen und technischen Literatur, Medienanalysen und Untersuchungen der "grauen« Literatur kombiniert, um die Konstruktion eines spezifischen Wissensregimes und seine Auswirkungen auf Gesellschaft nachzuzeichnen. Der Begriff Diskursanalyse für diese Art des Vorgehens ist dabei ein wenig irreleitend und gründet eher auf der theoretischen Nähe zu Foucault, als auf einer tatsächlichen methodischen Anwendung seines Ansatzes. Selten erreichen Forschungen eine Breite über verschiedene Felder und Domänen hinweg und eine historische Tiefe, die es rechtfertigen würden, von der Untersuchung eines Diskurses im Foucaultschen Sinne zu sprechen (vgl. Foucault 1981). 


\section{Ethnographische Methoden}

Einen zweiten methodischen Schwerpunkt bilden Forschungsansätze, die im weitesten Sinne als »ethnographisch« klassifiziert werden können. Im Kontext der Science and Technology Studies wird mit dem Adjektiv ethnographisch nicht notwendig auf die vielschichtige Karriere dieses Konzepts in der internationalen Sozial- und Kulturanthropologie angespielt, in der Ethnographie immer das Dreierlei aus Theorie-Empirie-Nexus, Feldforschung und Monographie bezeichnet. (ETHNOGRAPHISCHE PRAXIS) Vielmehr markiert die Selbstbeschreibung vieler Studien als ethnographisch lediglich methodische Vorgehensweisen, die teilnehmende Beobachtungen zur systematischen Datenerhebung einsetzen und damit über Interview-zentrierte Ansätze hinausgehen. Ethnographische Zugriffe auf Wissens- und Technologieproduktion etablierten sich gegen Ende der 1970er und frühen 1980er Jahre im Zuge der so genannten Laborstudien. (LABORSTUDIEN) Ethnographische Beobachtungen ermöglichten dabei einen Zugriff auf alltägliche wissenschaftliche Praxis, auf tat-sächliches Handeln wissenschaftlicher Akteure. Dies ist zunächst vor allem dort von Bedeutung, wo Formen von implizitem und verkörpertem Wissen für die Fragestellung eine Rolle spielen und daher Interviews zu kurz greifen, da sie lediglich die Untersuchung von explizierbarem und diskursivem Wissen ermöglichen.

Ferner bringen anthropologisch motivierte Laborstudien bereits früh das Argument in Anschlag, dass es sich bei wissenschaftlichen Gemeinschaften in Laboren oder Forschungszentren um Kollektive handele, die dem »Stamm« oder »der Kultur« der frühen anthropologischen Untersuchungen nicht prinzipiell unähnlich seien. Solche Studien betrachten wissenschaftliche Gruppen nicht primär als Expertengruppe, die hochgradig spezialisiertes Wissen produziert, sondern als ausdifferenzierte soziale Gemeinschaft, die unter spezifischen Bedingungen Alltag lebt. Ethnographisches Arbeiten im sozial- und kulturanthropologischen Sinne ist hier unerlässlich, denn es geht um die Kulturen von Laboren und den Habitus oder die Kosmologien von Forschenden, kurz um jene Handlungsorientierungen, die den Akteuren als »selbstverständlich« und unproblematisch erscheinen. Diese, »im Rücken der Akteure« wirkenden Orientierungen in Interviews zu erfragen, wäre daher zwecklos. Stattdessen verspricht der Blick »über die Schultern der Akteure« eine neue Perspektive. (Geertz 1973) Dies gilt ebenfalls für An- 
sätze, die nach der Reichweite wissenschaftlichen Wissens hinein in gesellschaftliche Alltage fragen.

Seite Mitte der 1990er Jahre nehmen Forschungen immer mehr diese Schnittfelder zwischen Wissenschaft und Gesellschaft in den Blick. Dabei geht die Entwicklung dahin, diese Schnittfelder nicht als Bereiche zu verstehen, in denen eine simple Übersetzung oder Popularisierung wissenschaftlichen Wissens für eine »Laien-Öffentlichkeit« stattfindet, wie dies etwa in manchen Ansätzen des public understanding of science bis in die 1990er Jahre überwiegend erfolgte. Vielmehr werden diese Schnittfelder als »Transaktionszonen« konzipiert und Interaktionen und Rückkoppelungs-Effekte zwischen gesellschaftlicher Ordnung und Wissens- und Technologieentwicklung unterstellt. (Vgl. Callon 1999; Hacking 2006; KLASSIFIKATIONEN) Um diesen vielschichtigen Interaktionen in gesellschaftlichen Alltagen nachzuspüren, sind ethnographische Methoden unerlässlich. Nur eine genaue Kenntnis der lokalen Praxen und Konfigurationen, der konkreten Aneignungspraktiken ermöglicht die Beobachtung und Analyse von langsamen und oft subtilen und indirekten Veränderungen. (Vgl. auch Hirschauer 1994) Gerade die unintendierten Nebeneffekte von Technologie- und Wissensentwicklung, die im Zuge der Reflexivisierung der Moderne seit spätestens den 1980er Jahren eine solch wichtige Rolle in Debatten um den Umgang mit Wissen und Technologie spielen, sind durch Diskurs-basierte Methoden nur begrenzt zu erfassen. (Beck 1986) Hier geht es vielmehr im klassischen Sinne um anthropologische Untersuchungen von sozialen und kulturellen Ordnungsprozessen und ihre Veränderungen unter Bedingungen von raschem Wissenszuwachs und technologischer Neuerung.

Allerdings darf bei allem sozialanthropologischem Enthusiasmus für ethnographische Methoden nicht außer Acht geraten, dass die Anwendung eines engen Methodenspektrums immer blinde Flecken mit sich bringt. Im Falle des ethnographischen Arbeitens liegen diese blinden Flecken vor allem darin, dass qualitativ-intensive Methoden nur die Analyse relativ kleiner, exemplarisch ausgewählter Untersuchungseinheiten erlauben. Ethnographische Methoden sind weniger gut geeignet, den strukturellen Rahmenbedingungen vieler Entwicklungen nachzugehen - hier sind etwa die Bourdieusche Feldanalyse oder historisch angelegte Untersuchungen, die längerfristige Zeiträume beobachten, Erfolg versprechender. Der genaue Blick auf den Alltag birgt also stets das Risiko einer präsentistisch vereng- 
ten Analyse; ein Problem, das gerade in den typischen Forschungsfeldern der Science and Technology Studies, die häufig durch langsame Verschiebungen statt durch abrupte Brüche gekennzeichnet sind, durchaus einige Brisanz besitzt.

\section{Praxeographie}

Praxeographie bezeichnet, ähnlich der Ethnographie, verschiedene Formen der teilnehmend beobachtenden Verfahren, ruht allerdings auf einem deutlich anderen theoretischen Fundament. Sie geht davon aus, dass soziale Phänomene, z. B. Menschen, Dinge oder Diskurse, sich nicht durch ein stabiles, ihnen innewohnendes, quasi essentielles Wesen auszeichnen, das dann in sozialer Praxis interagiert, sondern dass Phänomene immer nur so sind, wie sie in einer spezifischen Praxis gemacht werden. Nichts ist außerhalb von Praxis. (Vgl. DeLanda 2006; Mol 2002; Barad 1999) Praxis wird damit zur grundlegenden Untersuchungseinheit und nicht, wie in der Ethnographie, Akteure oder Strukturen. Dabei legt die Praxeographie Wert darauf, dass sie selbst immer notwendig Teil der Praxis ist, in der das zu untersuchende Phänomen produziert wird. (Mol et al. 2010) Die Forschenden sind also involviert, und dies hat nicht nur politische, moralische und epistemologische Konsequenzen, wie dies häufig für die Ethnographie diskutiert wird, sondern auch ontologische Konsequenzen für das zu untersuchende Phänomen. Ohne die Beteiligung einer Beobachterin wäre es anders, weil die das Phänomen (mit)produzierende Praxis anders wäre. Die Assoziation dieses Ansatzes mit der Praxistheorie des französischen Ethnologen, Soziologen und Kulturtheoretikers Pierre Bourdieu bleibt dabei implizit. (Bourdieu 1976) Bourdieu hat mit seiner Praxeologie eine Theorie entworfen, die weniger situativ und stärker auf die Analyse sozial differenzierter, stabiler Praxisformen in Gesellschaften gerichtet ist, während die Praxeographie eher dazu tendiert, Mikro-Praktiken detailliert zu analysieren. (weiterführend dazu der Abs. Praxis und Praxistheorie in diesem Kapitel)

\section{Governance und Steuerungsanalysen}

Ein dritter methodischer Schwerpunkt der Science and Technology Studies liegt auf der Analyse von politischen Prozessen im Bereich der Steuerung und Regulierung von Innovation, Wissenschaft und Technologieentwick- 
lung. (z. B. Hagendijk/Irwin 2006; Jasanoff et al. 1995) Hier orientieren sich Forschende vornehmlich an den organisations- und prozessanalytischen Methoden der Politik- und Sozialwissenschaften. Unter dem Stichwort governance geht es in diesem Teilgebiet der STS um ein besseres Verständnis der Entwicklungsdynamiken von Wissenschaft und Technologie und wie in diese Dynamiken mit spezifischen Zielvorstellungen interveniert werden kann. Ein wichtiges Ziel dieser Forschungsansätze ist es, den Wissensfundus und die Kompetenzen, die die Forschung in den Science and Technology Studies in den letzten dreißig Jahren erarbeitet hat, für wissenschafts- und hochschulpolitische aber auch wissensökonomische Entscheidungsprozesse fruchtbar zu machen.

Die Verfahren reichen hier von bibliometrischen und szientometrischen Forschungen zu Leistungs- und Qualitätsindikatoren in Wissenschaft- und Technologieentwicklung (Hornbostel 2006), über Akteurs- und Strukturanalysen von Innovationsinfrastrukturen bis hin zu politischen Analysen von Regulierungs- und Steuerungsprozessen vor allem unter Bedingungen von wissenschaftlicher Unsicherheit und Unwissen in Mehrebenensystemen. (Weingart 2002; Simon et al. 2001) Studien aus diesem Teilgebiet konzentrieren sich tendenziell auf die Makroebene gesellschaftlicher Entwicklung und verbinden sich nur in Ausnahmefällen mit Analysen von praktischem Alltag in der Wissensproduktion. Verbindungspunkte zu sozial- und kulturanthropologischen Perspektiven entstehen vor allem um Fragen nach den epistemologischen Konsequenzen spezifischer Rahmenbedingungen von Wissensproduktion. Dieser Art Fragen stellen allerdings eine methodische Herausforderung dar, da sie die Verbindung von makro- und mikroanalytischen Methoden notwendig machen. Umfassende Analysen dieser Art sind daher selten. 


\section{Die sozialanthropologische SPEZIFIK Dieser EINFÜHRUNG}

Es ist offensichtlich, dass diese Einführung ihre Akzente und Auslassungen vor deutlich disziplinärem Hintergrund vornimmt. Ein sozialanthropologischer Blick auf die Science and Technology Studies rückt zwangsläufig ethnographische Methoden in den Vordergrund, misst Praxis-orientierten Forschungsansätzen viel Raum zu und präferiert, wie im Folgenden deutlich wird, Problematisierungen mit einer sozial- und kulturanthropologischen Komponente. Auch die in diesem Band vorgenommene Zusammenstellung von Themen, die Auswahl der Beispielstudien und die selektive Darstellung von historischen Entwicklungslinien des Feldes der Science and Technology Studies ist als situierte Praxis zu interpretieren: als (fach-) diszipliniertes Tun, das vor dem Hintergrund mancher Kompetenz auch charakteristische blinde Flecke, Abneigungen und Ignoranzen offenbart.

\section{Drei Verschränkungen zwischen Sozialanthropologie und STS: Rationalität \& westliche Moderne}

Die Forschungen in den Science and Technology Studies untersuchen häufig Wissen und Technologie in ihren jeweiligen Konstruktionsprozessen. Was aber genau ist mit Konstruktion gemeint? Der kanadische Wissenschaftshistoriker und Philosoph Ian Hacking stellt zu Recht klar, dass die Aussage, dass wissenschaftliches Wissen das Ergebnis eines sozialen Prozesses darstellt, in vielerlei Hinsicht trivial ist. (Hacking 1999) Insoweit, als dies lediglich bedeuten soll, dass an seinem Entstehen Menschen beteiligt sind, die kommunizieren und interagieren, d. h. die in sozialen Prozessen kooperieren, hält die sozialkonstruktivistische Kritik wenig Aufregendes bereit. Ihr Verdienst liegt vor allem darin, wissenschaftliches Wissen und Technologie für sozialwissenschaftliche Analysen verfügbar gemacht und sie damit in Untersuchungen von Herrschaft und sozialer Ordnung eingebettet zu haben. Neben der bereits oben angeführten feministischen Kritik, ist dieser Schritt vor allem auch dem französischen Sozialtheoretiker und Philosophen Michel Foucault zu verdanken, der mit seinen mikropolitischen Analysen von Macht und Wissen den Weg für den Durchbruch des Sozialkonstruktivismus ebnete. (Vgl. Foucault 1974; Foucault 1978; Foucault 1980) 
Michel Foucaults Verständnis des Zusammenhangs von Diskurs, Wissen und Herrschaft hat das Feld der Science and Technology Studies entscheidend geprägt und wirkt bis heute in fast alle Teilbereiche der STS nach. Das Interessante und Wichtige an dieser Perspektive ist jedoch nicht oder zumindest nicht mehr, dass Wissen und Technologie das Ergebnis von sozialen Prozessen darstellen. Vielmehr geht es darum zu zeigen, wie genau diese Konstruktionsprozesse ablaufen, und wie sie daher spezifische Herrschaftskonstellationen reproduzieren oder verändern. Da dieses Wie für viele Forschende nicht nur menschliche Akteure wie Wissenschaftlerinnen, Ärzte oder Mediendesigner einschließt, sondern auch deren Techniken und Technologien, verkörpert und situiert in je spezifischen Apparaten und Konstellationen, sprechen heute viele Forschende in den STS nicht mehr von Sozialkonstruktivismus, sondern von Konstruktivismus, konstruktivistischem Realismus (Latour 2002; Barad 1999) oder PostKonstruktivismus (Lynch 1990; Kneer 2008), um deutlich zu machen, dass die Konstruktion von wissenschaftlichen Fakten nicht lediglich ein Ergebnis von - im ursprünglich soziologischen Sinne - sozialen Ursachenfaktoren ist, sondern eine Koproduktion aus Akteuren von unterschiedlichen ontologischen Status. (AKTEUR-NeTZwerk TheOrie und POSTANT) Post-konstruktivistische Analysen versuchen, die in vielen Bereichen der Sozial- wie Naturwissenschaften angelegten ontologischen Hierarchien nicht zu reproduzieren, sondern gehen stattdessen ontologisch »flach« zuwerke.

Mit dieser post-konstruktivistischen Perspektive gehen vor allem in der sozial-anthropologischen Lesart der STS, wie sie in diesem Band vorgestellt wird, eine Reihe weiterer wichtiger Verschiebungen in Bezug auf die Problematisierung von Rationalität, Materialität, Wissen und Praxis einher, auf die in den folgenden Abschnitten kurz eingegangen werden soll. Zunächst zur Frage von Rationalität und westlicher Moderne: Man hat dem Sozialkonstruktivismus zu Recht vorgeworfen, dass er einem problematischen Relativismus Tür und Tor geöffnet habe. Radikale Verfechter des Sozialkonstruktivismus argumentieren bis heute, dass wissenschaftliches Wissen als Ergebnis eines sozialen Konstruktionsprozesses sich in keiner Weise von anderen Wissensformen unterscheidet. Diese Position wird als Relativismus bezeichnet, weil wissenschaftliches Wissen damit seine Rückbindung an Absolutheit wie Wahrheit oder Realität verliert und nur noch relativ zu seinen Konstruktionsbedingungen verstanden werden kann. Diese 
radikale Position hat durchaus ihre Berechtigung, erlaubt sie den Forschenden die Interessen und Machtverhältnisse innerhalb des wissenschaftlichen Feldes in den Vordergrund zu rücken.

Sie misst aber einer wichtigen Einsicht wenig Bedeutung bei, die bereits Foucault hatte. Aus Foucaultscher Sicht ist Wissenschaft vor allem die Produktion eines Diskurses - oder zumindest in vielen Feldern ein wichtiger Beitrag dazu -, der bestimmt, was als wahr gilt und was nicht. Wahrheitsdiskurse sind jedoch nicht einfach zu produzieren und zu stabilisieren. Vor allem in hochgradig ausdifferenzierten westlich-modernen Gesellschaften braucht es eine Menge Arbeit verschiedenster Art, um eine hochgradig stabile Wahrheitsproduktionsmaschine wie die Natur- und Ingenieurswissenschaften am Laufen zu halten. »Wahrheiten« in vielen anderen Feldern abseits der Wissenschaft sind von deutlich flüchtigerer Natur. Sie bestehen in recht spezifischen, gesellschaftlichen Konstellationen und vergehen, sobald sich diese Konstellationen verändern. Die Naturwissenschaften jedoch spielen seit der europäischen Aufklärung, also etwa seit dem späten 17. Jahrhundert, eine zunehmend wichtigere Rolle in gesellschaftlichen Ordnungsprozessen.

Im Laufe des 20. Jahrhunderts und vor allem seit dem 2. Weltkrieg haben sie eine derart dominante Position eingenommen, dass viele Kommentatorinnen und Kommentatoren gesellschaftlicher Entwicklung bereits das Wirken einer Expertokratie bemängeln. (STS UND POLITIK) Damit problematisieren sie den Grad, bis zu dem Entscheidungen, die lange Zeit als politisch-moralische Entscheidungen galten, die eine Gesellschaft zu treffen hat, nun beinahe ausschließlich durch Expertengremien getroffen werden, die mit wissenschaftlichen Spezialisten ${ }^{4}$ besetzt sind. Hier zeigt sich nicht nur die unglaubliche Dominanz naturwissenschaftlichen Wissens, sondern auch die Unanfechtbarkeit des Wahrheitsdiskurses, den diese Wissenschaften produzieren. Naturwissenschaftliche Erklärungen sind heutzutage derart plausibel, dass es schon fast nicht mehr auffällt, dass es sich nur um ein Erklärungsmodell von Welt handelt - und ein hochgradig spezifisches noch dazu.

An dieser Selbstverständlichkeit von naturwissenschaftlichen Wahrheitsdiskursen setzt die sozialanthropologisch orientierte Forschung in den Science and Technology Studies an. Sie macht sich ihren vergleichenden

\footnotetext{
4 Die rein maskuline Form ist hier durchaus intendiert.
} 
Blick und ihren kulturvergleichenden Theoriekorpus zu Nutze, um naturwissenschaftliches Wissen als eine kontingente, westliche, moderne und hochgradig rationale Form von Wissen im Blick zu behalten. (RATIONALIТÄт) Dies ist kein relativistisches Argument. Es geht keineswegs darum, Naturwissenschaft mit anderen Wissensformen gleichzusetzen, auch wenn es natürlich einen sozial und kulturell geprägten Alltag von wissenschaftlichem Handeln gibt. Es geht vielmehr um die Spezifik dieser Alltage. Ein molekularbiologisches Labor heutzutage hat in vielerlei Hinsicht sehr wenig gemein mit subsaharischem Schamanismus oder vernakularem Alltagswissen in den Subkulturen westlicher Metropolen. Wissenschaftliche Wissensproduktion muss also auch als solche, in ihren je spezifischen Eigenheiten und Alltagen, beschrieben und analysiert werden.

Die entscheidende Frage aus sozialanthropologischer Sicht ist jedoch: wie trägt dieses Wissen als Praxis im Alltag zu gesellschaftlichen Ordnungsprozessen bei? Auf dieser Ebene geht es um die Reichweite und die Wirkweisen wissenschaftlichen Wissens hinein in alle möglichen Alltage. Es geht um die Art und Weise, wie dieses Wissen Alltage erreicht, z. B. als verschriftlichtes Wissen in populären Medien, als materialisiertes Wissen in technologischen Neuerungen oder als Praxiswissen in den Behandlungen durch Spezialisten und Spezialistinnen, bspw. in der Medizin. Es geht um Aushandlungsprozesse zwischen verschiedenen Wissensformen, um konkrete, praktische Interaktion und die Vermittlung von Sprache, Technologien, Repräsentationen usw. in Alltagspraxis. In allen Fällen mischt sich wissenschaftliches Wissen in der Praxis mit bereits existierenden alternativen und häufig konkurrierenden Ordnungsansprüchen.

Spätestens seit den Arbeiten des britischen Sozialanthropologen E.E. Evans-Pritchard bei den Azande im heutigen Sudan, in den späten 1930er Jahren, sind die damit aufgerufenen Fragen zentrale Arbeitsfelder der Anthropologie. Wie erklärt sich eine spezifische Gruppe von Menschen bestimmte Ereignisse? Welche Wissensformen werden dabei mobilisiert von wem und in welcher Art und Weise? Evans-Pritchard hat mit seiner Studie der Rolle von Magie bei den Azande hier eine wichtige Perspektive eröffnet. Anhand eines Kornspeichers, der kollabiert und dabei eine Reihe von Menschen unter sich begräbt, stellt er die Frage, wie dieses Ereignis bei den Azande erklärt wird. Während sich durch die westliche Moderne geprägte Beobachter damit zufrieden geben, dass das Holzgebäude wohl durch Termiten zum Einsturz gebracht wurde, ist dies für die Azande keine ausrei- 
chende Erklärung. Sie wollen auch erklären, warum es genau zu diesem Zeitpunkt zusammenfiel und bestimmte Menschen - und nicht andere - tötete. Was für westliche Betrachter durch den Verweis auf einen »Zufall« wegerklärt wird, interpretieren die Azande als das Ergebnis eines Zaubers wohlgemerkt: den Zeitpunkt, nicht die Tatsache des Einsturzes. (RATIONALITÄT)

Hier wird deutlich, dass westliche, modern-naturwissenschaftliche Vorstellungen von Kausalität, von Rationalität und von Reduktionismus nur einen möglichen Erklärungsansatz darstellen, der dabei immer mit alternativen Erklärungsmöglichkeiten konkurriert. Selbstverständlich nimmt die naturwissenschaftlich-rationale Erklärung in westlichen Gesellschaften eine zentrale Rolle ein. Aber es ist und bleibt eine wichtige Aufgabe der sozialanthropologischen Science and Technology Studies immer wieder die Kontingenz dieses Zugriffs auf Welt vor Augen zu führen, d. h. mittels empirischer Methoden und epistemologischer Analysen zu zeigen, dass diese Art der Erklärung eine mögliche, aber keinesfalls die notwendige ist; und zu zeigen, wie diese Art der rationalen Erklärung mit alternativen Erklärungen um Plausibilität, Nützlichkeit und Wirkmacht streitet. Letztlich ist mit diesem Ansatz die Frage formuliert, in welcher Art und Weise Wissenschaft gesellschaftlichen Alltag formt, ordnet und auf spezifische Art und Weise stabilisiert. Und die gleiche Frage wird natürlich auch in die andere Richtung gestellt, nämlich: wie ist Wissenschaft als Alltagspraxis vielfach situiert. Wie macht sich bemerkbar, dass Wissenschaft ein integraler Bestandteil von Kultur ist und wie reflektieren und reproduzieren die Ordnungsansprüche von Wissenschaft immer auch politische Werte, wirtschaftliche Prioritäten oder kulturelle wie kosmologische Konfigurationen?

\section{Materielle Kultur \& Körperlichkeit}

Materialität hat in der Sozial- und Kulturanthropologie stets eine wichtige Rolle gespielt. So widmete sich die Sachkulturforschung der deutschsprachigen Volkskunde der Analyse von materiellen Artefakten und die internationale Anthropologie entwickelte das Feld der material culture studies. Von Seiten der Anthropologie hört man daher oft, dass die Behauptung der Science and Technology Studies, ihre Besonderheit liege nicht zuletzt darin, Materialität ernst zu nehmen, vor allem der Unkenntnis der Fachgeschichte der Anthropologie seitens der STS Gemeinde geschuldet sei. Dies ist ein fundamentales Missverständnis. Zwar gibt es einige wichtige Parallelen in 
der Art und Weise wie Anthropologie und Science and Technology Studies mit Materialität umgehen. Auch hat vor allem die kulturanthropologisch orientierte Forschung innerhalb der STS viel zum jetzigen Umgang mit Materialität in den Science and Technology Studies beigetragen. Jedoch ignoriert die Behauptung, dass die Anthropologie seit jeher Materialität in ihren Analysen berücksichtigt habe und STS in dieser Hinsicht nichts Neues zu bieten hätte, die Entwicklungen in der feministischen Anthropologie und der Akteur-Netzwerk Theorie der letzten zwanzig Jahre. Denn in diesen Studien wurde vor allem die traditionelle Subjekt-Objekt Unterscheidung problematisiert und weitgehend aufgehoben. Damit wurde Materialität gänzlich neu als Akteur im Alltagshandeln konzipiert. Diese Entwicklung ist besonders fruchtbar gewesen in Studien zu neuen Formen von Körperlichkeit und Verkörperungen (embodiment).

Zunächst jedoch zur Rolle von Materialität in der anthropologischen Fachgeschichte: Die Thematisierung materieller Kultur hat in der Anthropologie eine lange Geschichte, die sogar noch bis in ihre Gründungsphase reicht. Einige der Wurzeln der heutigen Kulturanthropologie US-amerikanischer Ausrichtung liegen etwa in der deutschen Anthropogeographie der Romantik, die zu Beginn des 19. Jahrhunderts wesentlich durch die Brüder Humboldt geprägt wurde. Anthropogeographie in der damaligen Lesart bezeichnete ein Forschungsprogramm, dass die Entwicklung von Völkern in enger Verbindung mit den Bedingungen in ihrer materiellen Umwelt, d. h. ihrer Ökologie, untersuchte. Hier wird also schon in den Geburtsstunden der Kulturanthropologie Materialität wesentlich für die Analyse von menschlicher Entwicklung; jedoch nicht in Form von Artefakten, d. h. als Symbolträger, sondern im Sinne der physischen Umwelt von menschlichen Gruppen, die nicht getrennt von, sondern als konstitutiv für einen Volksgeist oder Nationalcharakter zu betrachten sei. Das, was die Anthropologie dann kurze Zeit später weniger romantisch als Kultur einer spezifischen Gruppe bezeichnete, spielte sich also immer in einer materiellen Umwelt ab, die es mit zu berücksichtigen galt.

Franz Boas, einer der geistigen Väter der amerikanischen Kulturanthropologie, trug wesentlich dazu bei, diese Perspektive auf Menschen in ihrer Umwelt weiter zu entwickeln und dabei Materialität nicht aus dem analytischen Blick zu verlieren. Boas konzipierte und beforschte neben der materiellen Umwelt den Menschen selbst immer auch als physischen Körper, d. h. in seiner Materialität. Zwar wandte er sich rasch und sehr bewusst von 
der Idee ab, dass menschliche Entwicklung und menschliches Zusammenleben Gesetzmäßigkeiten im Sinne von Naturgesetzen folge, die man entschlüsseln könne; eine Position, wie sie Ende des 19. Jahrhunderts vor allem die Verfechter evolutionistischer Ansätze vertraten. Jedoch konzipierte er den Menschen in seiner historisch spezifisch situierten Weise immer auch als biologisches und nicht nur als soziales oder kulturelles Wesen, ähnlich, wie das Vertreter der STS später forderten. Und seine empirischen Methoden zielten fast immer ab auf ein historisches Verständnis der konkreten Lebensbedingungen einer Gruppe von Menschen und den Versuch, Wirkungen dieser Lebensbedingungen auf physiologische Parameter dieser Gruppen zu messen. (Vgl. Boas 1922) Materialität bezog sich also in dieser frühen Form der Kulturanthropologie nicht nur auf »natürliche« Umwelt, sondern immer auch auf Körperlichkeit bzw. Physiologie in seiner spezifischen, historisch-kulturellen Ausformung.

In seiner dritten, und im 20. Jahrhundert sicherlich dominantesten Form, tritt Materialität in der Kulturanthropologie in Form von Untersuchungen von Artefakten auf. Viele der hochgradig ritualisierten Praxisformen, die die Anthropologie der 1930er, 40er und 50er Jahre in den »primitiven« Kulturen untersuchte, beziehen materielle Artefakte in ihre Abläufe ein. Als prominentes Beispiel könnte man hier an den Totemismus denken, bei dem verschiedene Artefakte - oft aufwendige Holzschnitzereien symbolisch aufgeladen werden und etwa die Ahnen der jeweiligen Gruppe verkörpern und in relevanter Weise in Ritualen präsent machen. In ähnlicher Weise untersucht die Sachkulturforschung der deutschen Volkskunde beispielsweise den Aufbau einer typischen süddeutschen Schreinerwerkstatt als Repräsentant für eine spezifische handwerkliche Tradition. (SOzIALE Konstruktion von Technologie) Die Dinge können hier als Repräsentanten einer Kultur gelten, da ihr Gebrauch sie über die Jahre mit den Eigenheiten dieser Kultur aufgeladen hat. Materialität wird also vornehmlich als Speicher für Kultur verstanden, der durch die Analyse von materieller Kultur ausgelesen werden kann. Eine ganz ähnliche Perspektive auf Materialität findet sich auch in der französischen Kulturtheorie eines Pierre Bourdieu und seinem Konzept der verkörperten Wissens; hier allerdings in einer praxistheoretisch unterfütterten Lesart, auf die in den folgenden $\mathrm{Ab}$ schnitten noch näher eingegangen wird.

Zumindest der US-amerikanischen Kulturanthropologie ging mit der Wende zur interpretativen oder deutenden Anthropologie in den 1970er 
Jahren dieser Zugriff auf Materialität weitestgehend verloren. Clifford Geertz' Konzept der dichten Beschreibung richtete anthropologische Analysen auf die Erfassung von Bedeutung aus, auf das Lesen von Kultur als Text. (Geertz 1973) Dichte Beschreibung bezeichnet im Kern das Nachvollziehen der Beschreibungen eines Phänomens aus verschiedenen, lokalen Perspektiven durch die Ethnographin bzw. den Ethnographen. Damit rückten Sinn, Bedeutung und Text in den Vordergrund der Analyse und verdrängten die materiellen Aspekte von Kultur und von menschlichem Zusammenleben; eine Entwicklung, die die britische Sozialanthropologie nie in diesem Maße nachvollzogen hat. (z. B. Bloch 2005; Ingold 2000; Edwards 1995)

In den späten 1980er und 1990er Jahren wandelt sich die Rolle von Materialität im Schnittfeld von Kulturanthropologie und Science and Technology Studies in signifikanter Art und Weise. Dies hatte erstens mit den feministisch kritischen Ansätzen zu tun, wie sie vor allem die amerikanische Kulturanthropologin Donna Haraway bis heute vertritt. (Vgl. Haraway 1984; Haraway 1988) Sie prägte das Konzept der materiell-semiotischen Praxis und verknüpfte damit unauflöslich Dinge und Bedeutung. Bedeutung existiere niemals abseits von materieller Form, so Haraway, und das Materielle erlange erst spezifische Form durch die Verknüpfung mit Bedeutung. Diese beiden Bewegungen sind kennzeichnend für jede Form von Praxis und Alltag. Materialität verliert damit seine Rolle als träges Speichermedium von Kultur, das nur so von Bedeutung für das Leben der Menschen ist, wie es von diesen mit Bedeutung aufgeladen wird. Haraway insistiert, dass Praxis abseits ihrer materiellen Formen zu untersuchen, schlichtweg keinen Sinn mache. Sie beginnt also, Materialität gegenüber der Bedeutung aufzuwerten.

Anders theoretisch hergeleitet, doch im Ergebnis sehr ähnlich, argumentieren der französische Ethnologe, Soziologe und Sozialphilosoph Bruno Latour und seine Kolleginnen und Kollegen beim Entwurf der Akteur-Netzwerk Theorie. (Latour 1988) Sie lösen die aus ihrer Sicht spezifisch moderne Trennung von Natur und Kultur auf und beginnen, der frühen Anthropologie nicht unähnlich, Beziehungen zu analysieren; Beziehungen sowohl zwischen Menschen, als auch zwischen Menschen und Dingen. Menschliches Zusammenleben wird nun nicht mehr nur aus Sicht der menschlichen Akteure erklärt, sondern durch das netzwerkartige, sich ständig verändernde Beziehungsgeflecht zwischen Menschen und Dingen. (AKteur-Netzwerk Theorie) Materialität wird, in dieser Perspektive 
noch stärker als bei Haraway, zum Akteur und leistet einen aktiven Beitrag bei der Ausformung von sozialen Phänomenen, die dementsprechend als sozio-materielle, bzw. hybride Phänomene bezeichnet werden. Diese Lesart, der sehr aktiven Rolle von Dingen, macht vor allem in Analysen von naturwissenschaftlichen Feldern viel Sinn, da Forschungsfelder wie z. B. die Molekularbiologie in den letzten fünfzig Jahren ganz entscheidend immer auch durch die Rolle von Technologie geprägt wurden. So liegt es nahe, eine DNA-Sequenziermaschine in der Genomik nicht nur als materiellen Speicher biologischen Wissens zu verstehen, sondern sie ebenfalls als aktiven Akteur in die Analyse der Entwicklungen von genetischen Experimenten einzubeziehen. Soziologisch formuliert, überantwortet diese Form der Analyse den Maschinen und den Dingen Handlungsträgerschaft.

Eine solch aktive Rolle hat die Anthropologie der ersten Hälfte des 20. Jahrhunderts den Dingen niemals zugedacht. Sicherlich kann man darüber streiten, ob es nicht doch ein wenig zu viel französischen Chics ist, Dinge zu aktiven Akteuren zu machen. Schließlich scheint es auch sehr plausibel anzunehmen, dass es letztlich doch immer wieder der Mensch ist, der die Dinge aktiv werden lässt und der bestimmt, wie sie in Handlungsabläufe eingebunden werden. Diese Debatte ist lange und hart geführt worden und setzt sich bis heute fort. (SOCIOLOGY OF SCIENTIFIC KNOWLEDGE) Unabhängig von der epistemologischen Position, die man in diesem Feld beziehen möchte, ist jedoch unstrittig, dass feministische Kritik und AkteurNetzwerk Theorie einen neuartigen, relationalen Blick auf Forschungsfelder erwirkt haben, der die Rolle von Dingen, von Technologien und von Artefakten in neuen Zusammenhängen problematisiert und dass hinter diese theoretischen Entwicklungen kein Zurückgehen möglich ist. (Vgl. auch Effekte der writing culture Debatte auf Ethnographie als Text: ETHNOGRAPHIE)

\section{Praxis \& Praxistheorie}

Der Begriff der Praxis hat in der sozial- und kulturanthropologischen wie der soziologischen Forschung eine lange Tradition, wobei der Begriff in wechselnden Ausprägungen Verwendung fand; deshalb ist nicht einfach zu fassen, was mit diesem Konzept gemeint ist. Eine erste Annäherung: stellt man sich die Frage, wie Menschen in Gruppen zusammenleben, so wie dies Anthropologie und Soziologie häufig tun, so nehmen die Antworten verschiedene Dimensionen in den Blick. Eine dieser Dimensionen hat mit 
Ordnungsprozessen zu tun, d. h. mit der Beobachtung, dass menschliches Zusammenleben häufig in geregelten Bahnen verläuft. Antworten auf die Fragen, woher diese Bahnen kommen, wie sie wirken, wo sie gespeichert und wie sie reproduziert werden, fallen dabei unweigerlich in ein Spektrum, dessen entgegengesetzte Endpunkte auf der einen Seite von individualistischen und auf der anderen von kollektivistischen Konzepten gebildet werden. Dabei können abhängig vom Denkstil für den individualistischen Endpunkt Begriffe wie Hirn, Geist, Akteur oder Subjekt einstehen, während auf der kollektivistischen Seite häufig von Struktur, Gesellschaft oder Klasse gesprochen wird. Zentraler wissenschaftlicher Streitpunkt mit hoher politischer Sprengkraft ist dabei die Frage, inwieweit ein Individuum sein eigenes Handeln selbstbestimmt zu steuern vermag bzw. bis zu welchem Grad menschliches Handeln durch den jeweiligen sozialen und materiellen Kontext vorgegeben ist. Denn weder die Annahme völliger individueller Freiheit, noch die Annahme, dass Menschen lediglich vorgegebene »Programme« realisieren, scheint empirisch überzeugend begründbar.

Das Konzept der Praxis steht nun zwischen diesen beiden Endpunkten. Praxis bezeichnet das Wie konkreten lokalen menschlichen Zusammenlebens und begreift dieses als Ergebnis des Zusammenwirkens kollektivistischer und individualistischer Elemente. Dem Praxisbegriff stehen die Konzepte Handlung, Praktik und Alltag nahe, die häufig auch synonym verwendet, bzw. unsauber voneinander abgegrenzt werden. ${ }^{5}$ Dies gilt im besonderen Maße für den Alltagsbegriff der Sozial- und Kulturanthropologie. Dies liegt vor allem daran, dass die Anthropologie Alltag meist als Gefüge von Routinen versteht, d. h. als ein in geregelten Bahnen verlaufendes Mit-

5 Allerdings kann man zumindest Tendenzen in der Verwendung ausmachen, mittels derer diese Begriffe abgegrenzt werden können. Handlung bezeichnet in der Regel ein individuelles Tun, das erstens auf einen mit Motivation und Intention ausgestatteten Akteur zurückzuführen ist, und zweitens diesem in einem spezifischen sozialen Kontext als sinnhaft erscheint. Der Begriff der Praktik, wenn er denn überhaupt von Praxis unterschieden wird (vgl. practice im Englischen), wird eher in Bezug auf ein klar umrissenes Handlungsgefüge verwendet und steht häufig in Bezug zu spezifischen individuellen Fähigkeiten. So würde man im Zusammenhang mit den Abläufen in einem medizinischen Operationssaal wohl von den Praktiken des Operierens sprechen, wenn man die individuellen Abläufe meint, die eine Operateurin zu meistern hat, aber von der Praxis des Operierens, wenn es um den Operationsstil z. B. eines spezifischen OP Teams ginge. Die Trennung ist alles andere als scharf. 
einander von Menschen in spezifischen Settings. Wenn man Praxis und Alltag unterscheiden möchte, dann am ehesten so, dass Alltag vor allem solche Praxen meint, die im Allgemeinen durch Normalität, Routine und Gewohnheit bestimmt sind, wohingegen der Praxisbegriff diese Verengung nicht notwendig mit sich bringt.

So unscharf diese Begriffe sein mögen, ergibt sich doch aus dem Praxisbegriff, wie er im Folgenden erläutert und situiert wird, eine spezifische Forschungsperspektive. Nämlich eine Perspektive, die auf überindividuelle, gleichsam habituelle Konfigurationen schaut, ohne eine deterministische Wirkweise dieser Konfigurationen im Sinne von Strukturen oder Gesetzmäßigkeiten zu postulieren; die materielle und soziale Elemente symmetrisch berücksichtigt, statt die einen zu Vorbedingungen für die anderen zu machen; und die zwar Muster und Netzwerke rekonstruiert, aber immer noch größeres analytisches Interesse an ihren Dynamiken und Kontingenzen entwickelt. Praxis bezeichnet damit eine analytische Perspektive, die menschliches Zusammenleben als mannigfaltig situiert begreift. In der konkreten, ethnographischen Arbeit wird deshalb zwar dem direkt erfahrbaren Geschehen die größte Aufmerksamkeit gewidmet, darüber werden aber nie die historischen, materiellen und kulturellen Bedingtheiten eines jeden Geschehens aus den Augen verloren. Praxis bezieht damit verschiedene Zeithorizonte, verschiedene Räumlichkeiten und die materiell-gegen-ständlichen Kontexte in die Analyse des konkreten Wie menschlichen Zusammenlebens ein.

\section{Drei Stränge aus dem Rhizom der Praxistheorien ${ }^{6}$}

In der Sozial- und Kulturanthropologie hat der Praxisbegriff durch das gesamte 20. Jahrhundert mehr oder minder explizit eine zentrale Rolle gespielt. Die Interaktivität ethnographischen Forschens, die Beschäftigung mit dem Alltag fremder Kulturen und die Zusammenführung verschiedener analytischer Ebenen von individuellem Verhalten bis hin zu Kosmologien, waren immer schon Problematisierungsformen, die zumindest mit impliziten Praxisbegriffen operiert haben. Allerdings standen in der anthropologischen Forschung der 1950er - 1970er Jahre lange Zeit andere Konzepte im Vordergrund.

6 Die folgenden beiden Abschnitte zu Bourdieu und Regelmäßigkeit/Kontingenz sind in wesentlichen Zügen entlehnt aus Beck (1996). 
Sherry B. Ortner, amerikanische Kulturanthropologin, skizziert die Entwicklung wie folgt (Ortner 1984; Sahlins 1993): Ende der 50er Jahre seien die »erschöpften« Theorien des britischen Strukturfunktionalismus (verbunden mit den »Gründungsvätern« einer akademisch respektablen Anthropologie in Großbritannien, A.R. Radcliffe-Brown und Bronislaw Malinowski), der amerikanischen Kulturanthropologie (wie sie etwa von Margaret Mead oder Ruth Benedict vertreten wurde) und der amerikanischen evolutionistischen Anthropologie (wie sie etwa Leslie White und Julian Steward konzipiert hatten) durch drei mit viel Aggressivität und Innovationskraft platzierte theoretische Bewegungen einer jüngeren Generation von Anthropologen abgelöst worden: (a) die symbolisch-interpretative Anthropologie von Clifford Geertz und Victor Turner, (b) die Kulturökologie, ausgearbeitet etwa von Marshall Sahlins oder Roy Rappaport, und schließlich (c) durch die Rezeption der in Frankreich dominierenden strukturalen Anthropologie von Claude Lévi-Strauss'. (Vgl. Sahlins 1993; Lévi-Strauss 1968; Geertz 1973)

Diese drei Bewegungen bergen in und zwischen sich vielfältige Spannungen, von denen diejenige zwischen idealistisch, symboltheoretischinterpretativen einerseits und materialistischen Perspektiven andererseits wohl die offensichtlichste darstellt. In dieser Konstellation der frühen 1970er Jahre bietet die neue Orientierung hin auf die Problematisierung von Praxis, Handeln oder - allgemeiner - der Tätigkeit von gesellschaftlichen Akteuren eine explizite Schlichtungsgrundlage: Indem Ideologie ebenso wie die materiellen Bedingungen sozialen Lebens Berücksichtigung fanden, konnten Fragestellungen der symbolischen Anthropologie ebenso aufgegriffen werden wie die Problemformulierungen der materialistisch argumentierenden Kulturökologie und im Rahmen einer Theorie interpretiert werden. Gleichzeitig wurden hierdurch wieder soziologische Kategorien in die amerikanische Kulturanthropologie eingeführt, was einen An-knüpfungspunkt an die Arbeiten der britischen Sozialanthropologie ermöglichte. Reichte bis zum Ende der 1970er Jahre noch der Einfluss des marxistisch inspirierten Strukturalismus als theoretisches Fundament für die Neuausrichtung der Forschung auf Praxis, so kommt diese Phase in der anthropologischen Theoriebildung spätestens zu Beginn der 1980er Jahre mit expliziten Praxistheorien, die sich deutlich von ihren Vorgängern absetzen, zu einem Ende. Eine zentrale Rolle spielte hier der französische Kultursoziologe und Ethnologe Pierre Bourdieu. 


\section{Pierre Bourdieu}

Bourdieu beklagt, die bisherigen Ansätze der strukturalen und symbolischen Anthropologie betrachteten Handeln stets nur »negativ, d. h. als Ausübung/Ausführung«. Diese Ansätze brächten verdinglichte Abstraktionen dank eines Fehlschlusses hervor, »der darin besteht, die von der Wissenschaft konstruierten Objekte wie >Kultur`, `Struktur`, >soziale Klassen`, >Produktionsweisen gesellschaftliche Wirksamkeit eignet«. (Bourdieu 1976) Kurz: der Fehler der Anthropologen bestehe darin, aus der Beobachtung der Muster konkreten Handelns abzuleiten, dass es irgendwo einen mächtigen, determinierenden Steuerungsmechanismus geben müsse - den sie dann als »Kultur« oder »Struktur« postulierten und der daraufhin ein theoretisches wie praktisches Eigenleben zu führen beginne. Alle diese Spielarten einer »fallacy of false concreteness« (Alfred North Whitehead) kritisiert Bourdieu als grundlegende Fehler einer objektivistischen Ethnologie, die eine wirkliche Theorie der Praxis verhindere:

»Die Theorie des Handelns als einer einfachen Ausübung des Modells ... stellt nur ein Beispiel unter anderen für jene imaginäre Anthropologie dar, die der Objektivismus erschafft, wenn er, in Marx' Worten >die Sache der Logik ^ für die `Logik der Sache` ausgebend, die objektive Bedeutung der Praxisformen und Werke zum subjektiven Zweck des Handelns der Produzenten dieser Praxisformen, Praktiken und Werke erhebt, mitsamt seinem unmöglichen homo oeconomicus, der seine Entscheidungen dem rationalen Kalkül unterwirft, seinen Akteuren, die bloße Rollen ausführen oder Modellen gemäß handeln«. (Bourdieu 1976: 159)

Bourdieu greift mit seiner Kritik der Praxisverständnisse der 1970er Jahre die bereits angesprochene Dimension zwischen Akteur und Struktur, zwischen Individuum und Kollektiv wieder auf und fügt ihr einen weiteren wichtigen Aspekt hinzu, nämlich den der Situativität. Um Praxis nicht einfach als Ausführung eines durch Strukturen vorgegebenen »Programms« zu verstehen, betont Bourdieu den situativen, improvisierenden und kreativen Charakter von Praxis. Er gesteht damit den Einzelnen deutlich mehr Freiheitsgerade und Möglichkeiten zu, innerhalb bestimmter Rahmenbedingungen und Situationen Formen des Zusammenlebens zu bestimmen, als dies bis dahin der Fall war. 
Als Ziel dieses Ansatzes fordert Bourdieu unter Verweis auf die FeuerbachThesen von Marx, dass eine Theorie der Praxis zu erarbeiten sei, »die die Praxis als Praxis konstituiert« (ebd.: 143); hierzu müssten alle Theorien aufgegeben werden, »die explizit oder implizit die Praxis zu einer mechanischen, durch die vorhergehenden Bedingungen unmittelbar determinierten Reaktionsform stempeln«. (ebd.: 169) Damit wird ein Rückgriff auf Konzepte wie Normen oder Rollen, die dem konkreten Handeln vorgängig sind, explizit ausgeschlossen. Ausgeschlossen werden muss jedoch gleichzeitig auch ein rein voluntaristisches Konzept des Handelns, mit dem ausschließlich der freie schöpferische Wille des Handelnden herausgestrichen würde. Bourdieu löst dieses zweifache Problem von ausreichender aber nicht überschießender Handlungsfreiheit durch eine doppelte Operation: einerseits durch die konzeptuelle Einführung des Habitus als einer verkörperten, die Praxis strukturierenden Struktur, mit dem dauerhafte Dispositionen und Geschmackspräferenzen erzeugt werden, andererseits durch die Berücksichtigung »der dialektischen Beziehung zwischen Dispositionen und Ereignis«. (Bourdieu 1976: 183) Hiermit betont Bourdieu den zentralen Stellenwert der jeweiligen Handlungssituation für die Praxen, in denen sich aus dem Spannungsverhältnis zwischen objektiven, situativ gegebenen Handlungsoptionen - einem ereignishaften, kontingenten Element - und dauerhaften, durch den Habitus erzeugten Handlungsdispositionen - dem strukturellen, statischen Element - eine »Konjunktur« ergebe, Handlungsumstände, die von den Handelnden genützt werden könnten. Praxis in diesem Sinne benötigt mithin zweierlei, wenn sie ans Licht treten soll: Situation und Disposition.

\section{Regelmäßigkeit und Kontingenz}

Bourdieu selbst hat seinen praxeologischen Anspruch in empirischen Studien allerdings nur bedingt eingelöst. Immer wieder kippt ihm der so wichtige Balanceakt zwischen Situativität und Disposition zu Ungunsten der situativen Faktoren in eine analytische Sprache, in der doch letztlich strukturelle Konzepte wie Ordnung und Klasse vorherrschen. Vor allem dieser Tendenz zur strukturalistischen Lesart von Praxis stellen sich in den späten 1980er Jahren eine Reihe von Autorinnen und Autoren entgegen, die die Situativität und vor allem die Unbestimmtheiten und Unsicherheiten von Praxis in den Vordergrund stellen. So schreibt beispielsweise die Anthropologin Sally Falk Moore: »Order never fully takes over, nor could it. 
The cultural, contractual, and technical imperatives leave gaps, require adjustments and interpretations to be applicable to particular situations, and are themselves full of ambiguities, inconsistencies, and often contradictions.« (Moore 1975: 220)

Neben den für die klassische Ethnographie typischen Fokus auf Regelmäßigkeit, Konsistenz und Übereinstimmung des Handelns mit »Ordnung « müsse daher »a focus on change, or process over time, and on paradox, conflict, inconsistency, contradiction, multiplicity and manipulability in social life« treten. (ebd.: 217) Unter einer akteurszentrierten Perspektive könnten somit zwei sich ergänzende Handlungsmuster untersucht werden: erstens der Versuch der Akteure $\gg$ to control their situations by struggling against indeterminacy, by trying to fix social reality, to harden it, to give it form and order and predictability« und zweitens gegenläufige Prozesse »by means of which people arrange their immediate situations $[\ldots]$ by exploiting the indeterminacies in the situation, or by generating such indeterminacies, or by reinterpreting or redefining the rules or relationships. « (Moore 1975: 234)

Im Gegensatz zu Bourdieu argumentiert Moore ausgehend von der Annahme unvollständiger, unsicherer Ordnung und Kontingenz. Hierdurch kann sie Ordnung als eine Strategie der Praxis analysieren: Ordnung stellt sich aus dieser Perspektive nicht als ein vorgängiges, alle Praxisformen bis ins letzte Detail prägendes, deterministisch wirkendes Konstrukt dar, sondern als ein von den Akteuren ausgehender, provisorischer und notwendig unvollständiger Stabilisierungsversuch des Sozialen. Diesem »ordnungsstiftenden« Akteur stellt Moore den Handelnden gegenüber, der günstige Gelegenheiten durchaus zu ergreifen weiß, indem er entweder Kontingenzen und Unbestimmtheiten in den gesellschaftlichen Ordnungssystemen im eigenen Interesse nutzt oder solche freien Räume zum eigenen Vorteil aktiv schafft. $^{7}$

7 Ein ganz ähnlicher Ausgleich zwischen Ordnung und Handlungsfreiheit findet sich in den Konzepten strategischen und taktischen Handelns bzw. der darin manifestierten Handlungstheorie des französischen Soziologen Michel de Certeau (1984) sowie in den soziologischen Entwürfen von Anthony Giddens (1984). 


\section{Praxiskonzepte heute}

In den letzten gut fünfzehn Jahren etablierte sich eine weitere Variante des Praxisbegriffs, der an der Mooreschen Lesart ansetzt, diese aber um eine wesentliche Dimension erweitert. Sowohl Marx als letztlich auch Moore, Giddens und de Certeau arbeiten mit einer scharfen Trennung von Subjekt und Objekt. In ihrem Denken und in ihrer Forschung existieren materielle Umwelten und in ihnen soziale und humane Akteure. Diese klare Trennung gerät Mitte der 1980er Jahre zunehmend unter Druck. Wie bereits im Abschnitt zu Materialität kurz angerissen, wird diese Trennung zum einen durch eine zunehmend elaborierte feministische Kritik aufgelöst, die vor allem in der US Amerikanischen Kulturanthropologie wichtige Resonanzen produziert. Zum anderen beginnt mit dem Ende der 1980er Jahre die Entwicklung der Akteur-Netzwerk Theorie vornehmlich in der europäischen Wissenschafts- und Technikforschung. Paradigmatisch für diese beiden Entwicklungsstränge stehen die Namen Donna Haraway und Bruno Latour. Beide entwickeln in praxistheoretisch orientierten Forschungsarbeiten Konzepte, die die herkömmlichen Grenzen von Objekt und Subjekt bzw. Natur und Kultur aufheben.

Haraway entwickelt das Konzept der materiell-semiotischen Praxis als eine neue fundamentale ontologische Einheit, die Materialität und Bedeutung notwendig und unwiederbringlich in Praxis miteinander verbindet. Das Eine ist nicht mehr ohne das Andere zu haben. Latour spricht in einer sehr ähnlichen Stoßrichtung, wenn auch aus einer deutlich anderen Motivation und Genealogie heraus, von sozio-technischen Netzwerken und konfiguriert damit »das Soziale« neu; nämlich nicht als präformierte Einheit, die materieller Praxis vorgeschaltet wäre, sondern als ontologisch mit Technik, mit Materialität und mit Körper Verbundenes. Das Akteur-Netzwerk, das zu Beginn der 1990er Jahre noch häufig als tatsächliches Netzwerk im Sinne von Knotenpunkten und Verbindungen verstanden wurde, ist rasch dynamisiert worden, so dass es schon Ende der 1990er Jahre vor allem um den Bindestrich in Akteur-Netzwerk geht und damit um die Frage, wie Akteure in spezifischen Konstellationen zueinander in Bezug gesetzt werden, wie diese Beziehungen stabilisiert werden und wie sie sich wieder lösen. (Post-ANT)

Die Marx'sche Trennung von Subjekt und Objekt, d. h. von Produktionsbedingungen und ihren Konsequenzen, »is too weak. [...] We need to think about agency - performance, doing things." (Pickering 2001: 163) 
Mit dieser aktuellsten Praxiswende werden nun also Handlungsträgerschaft und Kreativität verstanden als verteilt zwischen menschlichen und nichtmenschlichen Akteuren - Dinge handeln hier gleichsam mit, sie sind als »Aktanten« anzusehen. Im Vordergrund steht eine prozessuale Perspektive auf die Art und Weise, wie Beziehungen zwischen menschlichen und nichtmenschlichen Akteuren hergestellt werden. Der US Amerikanische Soziologe Theodore Schatzki fasst dementsprechend den neuen Praxisbegriff sehr weit: »By >practices $<$ I mean organized spatial-temporal manifolds of human activity.« (Schatzki 2010) Und noch eine weitere Radikalisierung des Praxiskonzepts folgt zu Beginn des 21. Jahrhundert. Die niederländische empirische Philosophin Annemarie Mol denkt die Implikationen der feministischen Kritik bzw. der Akteur-Netzwerk Bewegung konsequent zu Ende und formuliert daraus ein Praxisverständnis, bei dem Praxis die fundamentale analytische Einheit darstellt und aus ihr heraus überhaupt erst Akteure entstehen. (Mol 2002)

Mols Welt beginnt also weder mit Struktur noch Akteur, nicht mit Habitus oder Unsicherheit, sondern mit Praxis, mit Dingen, die sich ereignen, mit Tun, mit Alltag und mit Bewegung. Praxis wird, wie die amerikanische Anthropologin und Physikerin Karen Barad überspitzt formuliert (Barad 1999), gewissermaßen ontologisch primitiv, d. h. nur noch an sich selbst gebunden und der Existenz von Akteuren vorgeschaltet. Entitäten jeglicher Art, d. h. Menschen und Dinge, entstehen überhaupt erst aus Praxis heraus. Sie sind quasi zeitlich begrenzt eingefrorene, stabilisierte oder suspendierte Praxis. Sie kristallisieren sich aus Praxis heraus und erreichen dabei verschiedene Härtegrade. Entitäten, Menschen und Dinge verlieren aus dieser Sicht ein ihnen innewohnendes und unveränderliches Wesen. Sie sind immer nur so, wie sie in und durch eine spezifische lokale und kontingente Praxis gemacht werden. Und nur als solche lassen sie sich auch praxeographisch beforschen. Alles andere - Subjekt oder Identität - sind genau die Projektionen, die schon Bourdieu den Strukturalisten und Symbolisten vorgeworfen hat und die auch in der frühen feministischen Anthropologie einer Donna Haraway noch immer nicht völlig getilgt sind. 


\section{Wissen und Technologie als Praxis}

Das Forschungsfeld der Science and Technology Studies hat diese aktuellsten Entwicklungen entscheidend mit vorangetrieben. Laborforschung, feministische Anthropologien und Akteur-Netzwerk-Bewegung prägen die Genealogien, die die Sozial- und Kulturanthropologie eng mit einer ethnographischen Wissenschafts- und Technikforschung verschränkt. (Kontopodis et al. 2011) Es ist daher wenig verwunderlich, dass der Praxisbegriff, wie er hier eingeführt worden ist, für die sozialanthropologischen Science and Technology Studies eine elementare Rolle spielt. Er markiert die empirische wie analytische Perspektive. Wissen und Technologie, zwei zentrale Akteure in den Forschungsfeldern der Science and Technology Studies, werden niemals als wie auch immer präformierte, essentielle Entitäten begriffen. Wissen ist weder abstraktes Gut, noch mentalistisches Werkzeug oder lediglich Ergebnis rein kognitiver Prozesse. Und Technologie ist niemals nur Ding, Gebrauchsgegenstand oder Träger von symbolischem Gehalt im Sinne eines Artefakts.

Beide, Wissen und Technologie, werden im Forschungsfeld der sozialanthropologischen Science and Technology Studies immer als Teil von Praxis begriffen und beforscht. Das bedeutet nicht nur, dass sie in ihrem Alltagsgebrauch untersucht werden, auch wenn dieser eine wichtige Rolle spielt. Es bedeutet, dass Wissen und Technologie erst durch die lokalen Praxen in ihrem konkreten So-Sein bestimmt werden. Sie existieren nicht außerhalb von Praxis und können daher auch nur als Teil von Praxis untersucht werden. Dabei ist es häufig charakteristisch für naturwissenschaftliches Wissen und Technologien in westlichen Modernen, dass sie eine hohe Stabilität über verschiedene Praxisformen hinweg erreichen, d. h. sie produzieren Kontinuität. Diese Stabilität, z. B. einer wissenschaftlichen Methode, einer klinischen Diagnose oder eines Messinstruments, wird allerdings in der Praxis hergestellt; ihren Produktionsprozess gilt es mittels ethnographischer Forschung zu zeigen. Stabilität ist niemals vorausgesetzt durch die Forschungsperspektive. Sie ist keine Eigenschaft von Wissen oder Technologie. Sie ist immer Ergebnis spezifischer Praxis - und kann daher auch schnell wieder zusammenbrechen. Sie ist eine Regelmäßigkeit, die sich über verschiedene Praxen hinweg ergibt, und in keiner Weise ein Apriori, das Praxis determiniert.

Wissen ist also immer Wissenspraxis und als solche situiert, eingebettet, verortet, verkörpert und eingeschrieben. Gleiches gilt für Technologie: 
sie wird erst in konkreten Produktions- und Nutzungskontexten geformt. Und sie ist Akteur. Denn die hier vorgestellte Praxisperspektive geht nicht davon aus, dass Handlungsträgerschaft und Kreativität notwendig an die Intentionen menschlicher Akteure gebunden sind. Vielmehr werden Handlungsträgerschaft und Kreativität auf der Ebene von konkreter Praxis und damit als verteilt über menschliche und nicht-menschliche Akteure untersucht. Technologie kann in dieser Perspektive sehr wohl einen wichtigen Beitrag zur Ausformung spezifischer Praxisformen leisten. Ein solches Verständnis von Wissen und Technologie als Praxis macht auch deutlich, warum ethnographisches und praxeographisches Arbeiten eine so zentrale Methode für die sozialanthropologischen Science and Technology Studies darstellt. Es geht nicht nur um die Beobachtung von Alltagen und implizitem Wissen, sondern es geht in einem fundamentaleren Sinne um Praxis als analytische Ebene auf der Wissen und Technologie überhaupt erst auf relevante Weise geformt und bestimmt werden. Und Praxis in diesem Sinne ist über Diskurs und Sprache nur unzureichend bestimmt. Sie muss immer auch in ihrem konkreten Ablauf, ihrem Getan-Werden beobachtet werden.

Dabei sei an dieser Stelle nochmals angemerkt, dass diese Lesart von Praxis ein hohes Risiko birgt, präsentistisch zu forschen, d. h. das Beforschte aus sich selbst heraus zu erklären. Der Schlüssel zum Verständnis einer spezifischen Praxis wird dann nur in dieser Praxis selbst gesucht. Das ist nicht beabsichtigt. Zwar geht es nicht mehr primär darum, eine, wie auch immer geartete, Eigenlogik einer Praxis zu destillieren. Auch wird nicht, in einer Marx'schen Interpretation von Geschichte, davon ausgegangen, dass sich Praxis quasi automatisch reproduziert. Und vor allem betont praxeographisches Arbeiten die Rolle der Forschenden in der Koproduktion des Forschungsfeldes und der untersuchten Phänomene. Alle drei Einwände dürfen jedoch nicht darüber hinwegtäuschen, dass jede Praxis eine historische wie soziale und kulturelle Tiefe mit sich bringt. Zwar müssen alle Elemente, die eine Praxis formen, in jedem konkreten Moment aktualisiert werden, daraus folgt aber nicht, dass sie präsent und ethnographisch fassbar sein müssen.

Vieles bestimmt eine konkrete Praxis gerade durch seine Abwesenheit oder durch eine spezifische Form der Anwesenheit, die andere Formen der Anwesenheit verdrängt. Ein klassisches Beispiel, das diese Problematik veranschaulicht, ist die Krankengeschichte von Patienten. Sie ist natürlich relevant in der konkreten Situation der ärztlichen Konsultation. Allerdings 
muss sie dort nicht sichtbar sein. Sie ist in Akten, Hirnen und Daten gespeichert und beeinflusst so das Arzt-Patienten Gespräch als absent-present. (Law/Mol 2002) In anderen Situationen wird eine Krankengeschichte vielleicht auf vielfache Art und Weise präsent gemacht, z. B. in dem Ärztin und Patient gemeinsam die Krankenakte durchgehen, um über ein neues Medikamentenregime zu entscheiden. Praxis ist also selten nur aus dem heraus erklärbar, was in der konkreten Situation erfahrbar wird. Daher sollten zu ihrer Erforschung auch niemals nur ethno- und praxeographische Methoden herangezogen werden. Methoden, die es ermöglichen, eine Genealogie der Praxis und ihre Interaktion mit alternativen Ordnungsprozessen relevant zu machen, d. h. historische Analysen und mit einem weiteren Objektiv agierende anthropologische und soziologische Methoden, z. B. Feldanalysen, stehen gleichberechtigt neben Ethno- und Praxeographie und sollten, wo immer möglich, komplementär zum Einsatz kommen.

\section{Zum AufBAU diESER EINFÜHRUNG}

Die Science and Technology Studies stellen ein Forschungsfeld dar, das an deutschsprachigen Universitäten in der Lehre wesentlich weniger präsent ist als beispielsweise im Angloamerikanischen Raum. Allgemeine und umfassende Einführungen existieren deshalb bereits in englischer Sprache sowohl als Monographien, wie auch als breit aufgestellte und sehr renommiert besetzte Sammelbände. (Hess 1997; Hess 1995; Biagioli 1999; Jasanoff et al. 1995; Hackett et al. 2008) Der vorliegende Band ist allerdings keinesfalls eine bloße Übertragung dieser Werke ins Deutsche. Stattdessen präsentieren wir hier eine dezidiert sozialanthropologische Perspektive auf die Science and Technology Studies. Diese Perspektive ist einerseits eng an unsere eigenen Arbeiten im »Labor: Sozialanthropologische Wissenschaftsund Technikforschung« des Instituts für Europäische Ethnologie der Humboldt-Universität zu Berlin gebunden. Andererseits weist sie entscheidend darüber hinaus, stärkt ein international etabliertes Forschungsfeld und stützt damit eine Entwicklung, die institutionell in Deutschland nicht stark verankert ist. (Vgl. Kneer 2008) Wir hoffen, durch diesen auswählenden Zugriff weniger allgemein über STS sprechen zu dürfen, gerade dadurch aber den Blick immer wieder auf Themen, Zugriffe und Problematisierungen zu lenken, die auch für Studierende, Kolleginnen und Kollegen 
sowie Interessierte aus anderen Disziplinen und Feldern von Interesse sind, die sich bereits mit Wissenschafts- und Technikforschung befasst haben. Es sei aber an dieser Stelle dringend darauf hingewiesen, dass ein tieferer Einstieg in die hier eingeführten Themen und Problematisierungen immer der weiteren Lektüre auch in den jeweiligen Disziplinen bedarf. Wo es den Autorinnen und Autoren dieses Bandes von besonderer Notwendigkeit oder Nützlichkeit $\mathrm{zu}$ sein schien, haben sie auf weiterführende Texte und Zugänge explizit verwiesen.

Dieser Band ist in eine Einleitung und drei Hauptabschnitte gegliedert. Der erste Abschnitt umfasst die ersten beiden inhaltlichen Kapitel und beschäftigt sich mit den wissenschaftsphilosophischen und -soziologischen Grundlegungen sozialanthropologisch ausgerichteter Science and Technology Studies, namentlich der Wissenschaftstheorie (Kapitel 1) und der frühen Wissenschaftssoziologie (2). Es folgt der zweite Abschnitt mit den Kapiteln drei bis sieben, der die theoretischen Bewegungen und Forschungsansätze im Kern der Science and Technology Studies vorstellt: sociology of scientific knowledge (3), soziale Konstruktion von Technologie (4), Laborstudien (5) und Akteur-Netzwerk Theorie (6). Der dritte Abschnitt beschäftigt sich mit den Schnittfeldern zu sozialanthropologischen Kernthemen. Dabei steht in Kapitel sieben die Beziehung zwischen STS und Politik im Vordergrund. In Kapitel acht werden Fragen nach der Rolle verschiedener Rationalitäten in gesellschaftlichen Ordnungen diskutiert. Kapitel neun widmet sich der Ethnographie als Methode, Theorie-Methode-Nexus und Text und bezieht diese Diskussion auf Untersuchungen von Wissenschaft und Technologie in modernen Gesellschaften. Kapitel zehn widmet sich der Frage nach Klassifikationen und ihren Wechselwirkungen mit Selbstkonstitutions- und gesellschaftlichen Ordnungsprozessen. Kapitel elf konzentriert sich ebenfalls auf Wechselwirkungen zwischen Wissenschaft und Gesellschaft, untersucht diese aber mit einer Perspektive auf Infrastrukturen. Infrastrukturen residieren als analytisches Konzept zwischen Praxis und Struktur und ermöglichen vor allem eine genauere Untersuchung der materiellen Bedingungen von Wissensproduktion und Wissenstransfer. Der Band schließt in Kapitel zwölf mit einem Überblick über aktuelle Diskussionen und Theoriestränge, die sich im Zuge von Debatten um die Weiterentwicklung von Akteur-Netzwerk Theorie gebildet haben. Dies ist derzeit ein sehr aktives Forschungsgebiet und weist 
damit über diese Einführung hinaus in die aktuelle Literatur, wie sie sich in den einschlägigen Zeitschriften darstellt, wie z. B. in Social Studies of Science; Science, Technology \& Human Values; BioSocieties; Science, Technology and Innovation; Science Studies und vielen weiteren.

Allen Kapiteln ist eine kurze einführende Passage vorangestellt, die die wichtigsten Punkte des Kapitels zusammenfasst und gegebenenfalls in einen breiteren Kontext von methodischen und theoretischen Entwicklungen einfügt. Diese Einführungen sind in didaktischer Absicht verfasst und kristallisieren die take home message jedes Kapitels heraus. Dieser extremen Verkürzung steht eine Liste mit zwei oder drei weiterführenden Texten zur Seite, die den im Kapitel vorgestellten Ansatz ergänzen, vertiefen oder die Problematisierung in einer Studie tatsächlich zur Anwendung gebracht haben. Dabei sollen die Kurzzusammenfassungen dieser Texte lediglich der Orientierung dienen und keinesfalls als ausreichende Inhaltsangabe aufgefasst werden. Der Band verfügt über einen Personenund Sachindex, der bei der Orientierung und Erschließung von theoretischen Konzepten helfen soll. Der Band folgt zumindest in den Hauptteilen eins und zwei einer chronologischen Ordnung. Die Kapitel erheben aber den Anspruch alleinstehend lesbar und verständlich zu sein. Querverweise auf andere Kapitel sind durch den Kurztitel des Kapitels in Klammern und Kapitälchen gekennzeichnet.

\section{LITERATUR}

Barad, Karen (1999): »Agential Realism. Feminist Interventions in understanding Scientific Practices«. In: Mario Biagioli (Hg.), The Science Studies Reader, New York: Routledge, S. 1-11.

Beck, Stefan (1997): Umgang mit Technik: kulturelle Praxen und kulturwissenschaftliche Forschungskonzepte, Berlin: Akademie Verlag.

Beck, Stefan/Cil, Nevim/Hess, Sabine/Klotz, Maren/Knecht, Michi (2007):

Verwandtschaft Machen. Soziale Formen der Reproduktionsmedizin und Adoption in Deutschland und der Türkei, Sonderheft der Berliner Blätter - Ethnographische und Ethnologische Beiträge, Berlin: LITVerlag. 
Beck, Ulrich (1986): Risikogesellschaft. Auf dem Weg in eine andere Moderne, Frankfurt/M.: Suhrkamp.

Biagioli, Mario (1999): The Science Studies Reader, London/New York: Routledge.

Bloch, Maurice (2005): Essays on Cultural Transmission, London: Berg Publishers.

Bloor, David (1976): Knowledge and Social Imagery, Routledge Direct Editions, London/Boston: Routledge \& K. Paul.

Boas, Franz (1982/1922): Report on an anthropometric Investigation of the Population of the United States«. In: Franz Boas (Hg.), Race, Language and Culture, Chicago/London: University of Chicago Press, S. 28-59.

Bourdieu, Pierre (1976): Entwurf einer Theorie der Praxis auf der ethnologischen Grundlage der Kabylischen Gesellschaft, 1. Aufl, Frankfurt/M: Suhrkamp.

Callon, Michel (1999): »Some Elements of a Sociology of Translation. Domestication of the Scallops and the Fishermen of St.Brieuc Bay«. In: Mario Biagioli (Hg.), The Science Studies Reader, New York: Routledge, S. 67-84.

Collins, Harry M. (1975): »The Seven Sexes: A Study in the Sociology of a Phenomenon, or the Replication of Experiments in Physics«. Sociology 9(2), S. 205-24.

de Certeau, Michel (1984): The Practice of Everyday Life, Berkeley: University of California Press.

DeLanda, Manuel (2006): A New Philosophy of Society: Assemblage Theory and Social Complexity, London/New York: Continuum.

Edwards, Jeanette (1995): »Explicit Connections: Ethnographic Enquiry in North-West England«. In: Jeanette Edwards/Sarah Franklin/Eric Hirsch/Frances Price/Marily Strathern (Hg.), Technologies of Procreation. Kinship in the Age of Assisted Conception, Manchester: Manchester University Press, S. 42-66.

Foucault, Michel (1974): Die Ordnung der Dinge, Frankfurt/M.: Suhrkamp.

Foucault, Michel (1978): Dispositive der Macht. Über Sexualität, Wissen und Wahrheit, Berlin: Merve.

Foucault, Michel (1980): »Power/Knowledge: Selected Interviews and Other Writings 1972-1977«. In: Colin Gordon (Hg.), ebd., New York: Harvester Wheatsheaf. 
Foucault, Michel (1981): Archäologie des Wissens, Frankfurt/M.: Suhrkamp.

Geertz, Clifford (1973): The Interpretation of Cultures, New York: Basic Books.

Geertz, Clifford (1973): »Thick Description. Toward an interpretive Theory of Culture «. In: Clifford Geertz (Hg.), The Interpretation of Cultures: Selected Essays, New York: Basic Books, S. 3-30.

Giddens, Anthony (1984): The Constitution of Society: Outline of the Theory of Structuration, Cambridge/Cambridgeshire: Polity Press.

Habermas, Jürgen (1969): Technik und Wissenschaft als »Ideologie«, Frankfurt/M.: Suhrkamp.

Hackett, Edward J. (2008): The Handbook of Science and Technology Studies, Published in cooperation with the Society for the Social Studies of Science, Cambridge/MA.: MIT Press.

Hacking, Ian (2006): »Kinds of People: Moving Targets «. British Academy Lecture 10, S. 1-18.

Hacking, Ian (1999): The Social Construction of What?, Cambridge/MA: Harvard University Press.

Hagendijk, Rob/Irwin, Alan (2006): »Public Deliberation and Governance: Engaging with Science and Technology in Contemporary Europe«. Minerva 44(2), S. 167-84.

Haraway, Donna (1984): »Primatology Is Politics by Other Means «. PSA: Proceedings of the Biennial Meeting of the Philosophy of Sciences Association, Symposia and Invited Papers, S. 489-524.

Haraway, Donna (1988): »Situated Knowledges: The Science Question in Feminism and the Privilege of Partial Perspective«. Feminist Studies 14, S. 575-99.

Hess, David (1997): Science Studies - an Advanced Introduction, New York: New York University Press.

Hess, David J. (1995): Science and Technology in a Multicultural World. The Cultural Politics of Facts and Artifacts, New York: Columbia University Press.

Hirschauer, Stefan (1994): »Towards a Methodology of Investigations into the Strangeness of One's Own Culture: A Response to Collins«. Social Studies of Science. An International Review of Research in the Social Dimensions of Science and Technology 24, S 335-46. 
Hornbostel, Stefan (2006): »Leistungsmessung in der Forschung. Von der Qualitätssicherung der Lehre zur Qualitätsentwicklung als Prinzip der Hochschulsteuerung«. Beiträge zur Hochschulpolitik 1, S 219-28.

Ingold, Tim (2000): The Perception of the Environment: Essays on Livelihood, Dwelling \& Skill, London/New York: Routledge.

Jasanoff, Sheila/Markle, Gerald E./Petersen, James C./Pinch, Trevor (1995): Handbook of Science and Technology Studies, Thousand Oaks/Calif:: Sage Publications.

Kneer, Georg (2008): »Die Debatte über Konstruktivismus und Postkonstruktivismus«. In: Georg Kneer/Stephan Moebius (Hg.), Soziologische Kontroversen. Beiträge zu einer anderen Geschichte der Wissenschaft vom Sozialen, Frankfurt/M: Suhrkamp.

Kontopodis, Michalis/Niewöhner, Jörg/Beck, Stefan (2011): »Investigating Emerging Biomedical Practices: Zones of Awkward Engagement on Different Scales«. Science, Technology \& Human Values, online first.

Latour, Bruno (1988): "Mixing Humans and Nonhumans Togester: The Sociology of a Door-Closer«. Social Problems 35(3), S. 294-311.

Latour, Bruno (2002): Die Hoffnung der Pandora, Frankfurt/M: Suhrkamp.

Law, John/Mol, Annemarie (2002): Complexities: Social Studies of Knowledge Practices, Science and Cultural Theory, Durham: Duke University Press.

Lévi-Strauss, Claude (1968): Das wilde Denken, Frankfurt/M.: Suhrkamp.

Lynch, Michael/Woolgar, Steve (1990): Representation in Scientific Practice, Cambridge: MIT Press.

Mathar, Thomas (2010): »Der Digitale Patient. Zu Den Konsequenzen Eines Technowissenschaftlichen Gesundheitssystems«. Bielefeld: transcript.

Mathar, Thomas/Jansen, Yvonne J.F.M. (Hrsg.) (2010): »Health Promotion and Prevention Programs in Practice. How Patients' Health Practices are Rationalised, Reconceptualised and Reorganised «. Bielefeld: transcript.

Mol, Annemarie (2002): The Body Multiple: Ontology in Medical Practice, Durham: Duke University Press.

Mol, Annemarie/Moser, Ingunn/Pols, Jeannette (Hrsg.) (2010): »Care in Practice. On Tinkering in Clinics, Homes and Farms«. Bielefeld: transcript. 
Moore, Sally Falk (1975): »Epilogue: Uncertainties in Situations, Indeterminacies in Culture«. In: Barbara G. Myerhoff Sally Falk Moore (Hg.), Symbol and Politics in Communal Ideology. Cases and Questions, Ithaca/London: Cornell University Press, S. 210-39.

Nagel, Thomas (1986): The View from Nowhere, New York: Oxford University Press.

Niewöhner, Jörg/Kehl, Christoph/Beck, Stefan (Hrsg.) (2008): »Wie geht Kultur unter die Haut? Emergente Praxis am Schnittfeld von Medizin, Sozial- und Lebenswissenschaften«. Bielefeld: transcript.

Niewöhner, Jörg/Döring, Martin/Kontopodis, Michalis/Madarász, Jeannette/Heintze, Christoph (2011): „Cardiovascular Disease and Obesity Prevention in Germany: An Investigation into a Heterogeneous Engineering Project«. Science, Technology \& Human Values, online first.

Niewöhner, Jörg/Kehr, Janina/Vailly, Joelle (Hrsg.) (2011a): »Leben in Gesellschaft. Biomedizin - Politik - Sozialwissenschaften«. Bielefeld: transcript.

Ortner, Sherry B. (1984): »Theory in Anthropology since the Sixties«. Comparative Studies in Society and History 26(1), S. 126-66.

Sahlins, Marshall (1993): »Goodby to Tristes Tropes: Ethnography in the Context of Modern World History«. The Journal of Modern History 65(1), S. 1-25.

Schatzki, Theodore (2010): »Material and Social Life«. Nature and Culture $5(2)$, S. $123-49$.

Scheffer, Thomas/Niewöhner, Jörg (2010): Thick Comparison. Reviewing the Ethnographic Aspiration, Amsterdam: Brill.

Simon, Dagmar/Knie, Andreas/Hornbostel, Stefan (2001): Handbuch Wissenschaftspolitik. Wiesbaden: VS Verlag.

Weingart, Peter (2002): »The Moment of Truth for Science. The Consequences of the $>$ Knowledge Society< for Society and Science «. Embo Reports 3(8), S. 703-6. 